\title{
„Nun sag, wie hast du's mit den Geisteswissenschaften?““ Ein Review wissenschaftstheoretischer Grundlagen deutschsprachiger Lehrbücher der Allgemeinen Betriebswirtschaftslehre
}

\author{
Christian Julmi
}

Eingegangen: 16. Mai 2019 / Angenommen: 24. Januar 2020 / Online publiziert: 5. Februar 2020 (C) Der/die Autor(en) 2020

Zusammenfassung Obwohl die Wissenschaftspraxis in der Betriebswirtschaftslehre heute von einer methodologischen Offenheit geprägt ist, die quantitative und qualitative Forschung gleichermaßen für legitim erachtet, scheint die Allgemeine Betriebswirtschaftslehre immer noch einem einseitig-naturwissenschaftlichen Forschungsideal anzuhängen. Da die Allgemeine Betriebswirtschaftslehre die einheitliche Fundierung ihrer Disziplin anstrebt, stünde eine solche wissenschaftstheoretische Verengung im Widerspruch zu ihrer wissenschaftspraktischen Offenheit. In Bezug auf eine flächendeckende Verengung der Allgemeinen Betriebswirtschaftslehre auf ein naturwissenschaftliches Forschungsideal liegen bislang allerdings keine systematischen Untersuchungen vor. Das Ziel des Beitrags besteht daher in einem Review der wissenschaftstheoretischen Grundlagen der Allgemeinen Betriebswirtschaftslehre. Gegenstand des Reviews sind alle aktuellen, seit 2005 erschienenen deutschsprachigen Lehrbücher der Allgemeinen Betriebswirtschaftslehre. Im Ergebnis zeigt sich übereinstimmend, dass sich die Betriebswirtschaftslehre als anwendungsorientierte Geisteswissenschaft mit naturwissenschaftlichem Forschungsideal versteht und eine Verengung damit tatsächlich nachgewiesen werden kann. Im Anschluss an das Review stellt der Beitrag schließlich Überlegungen zu einer wissenschaftstheoretischen Revision der Allgemeinen Betriebswirtschaftslehre an, die dem praktizierten Methodenpluralismus entspricht und die praktische Relevanz verstehender Forschung würdigt.

Schlüsselwörter Wissenschaftstheorie · Praxisrelevanz · Verstehen · Hermeneutik · Systematisches Review

\footnotetext{
C. Julmi $(\bowtie)$

Fakultät für Wirtschaftswissenschaft, Lehrstuhl für Betriebswirtschaftslehre, insb. Organisation und Planung, FernUniversität in Hagen, 58084 Hagen, Deutschland

E-Mail: christian.julmi@fernuni-hagen.de
} 


\title{
"Tell me now, how do you feel about the humanities?" A review of the meta-theoretical foundations of German textbooks in general business administration
}

\begin{abstract}
Business administration today pursues a methodological openness in its research practice. Quantitative and qualitative research is considered equally legitimate. In contrast, general business administration in Germany still seems to adhere to a one-sided research ideal that only accepts the methodology of the natural sciences. Since general business administration strives for a uniform foundation of its discipline, this narrowing of scientific theory would not be compatible with its openness in scientific practice. However, there are no systematic studies available to date that prove an actual narrowing of general business administration to the methodology of the natural sciences. Therefore, this article reviews the meta-theoretical foundations of general business administration. Subject of the review are all German textbooks of general business administration published since 2005. The result shows unanimously that business administration is indeed an application-oriented discipline whose research ideal corresponds to the natural sciences. Following the review, the article discusses possibilities of a meta-theoretical revision in general business administration. Overall, the article argues for a methodological pluralism that recognizes the practical relevance of research in the humanities.
\end{abstract}

Keywords Scientific theory · Practical relevance - Theory of understanding · Hermeneutics $\cdot$ Systematic review

\section{Einleitung}

Hat sich die Betriebswirtschaftslehre lange Zeit ausschließlich an einem naturwissenschaftlichen Forschungsideal orientiert (z.B. Chmielewicz 1970), hat ab den 1970er Jahren eine Öffnung gegenüber geisteswissenschaftlichen Ansätzen stattgefunden, als deren prominenteste Vertreter Edmund Heinen mit seiner entscheidungsorientierten und Hans Ulrich mit seiner systemtheoretisch orientierten Betriebswirtschaftslehre gelten können (Schanz 2018, S. 60 ff.). Zu nennen ist zudem die konstruktive Betriebswirtschaftslehre von Steinmann und Kollegen, die auf der konstruktivistischen Philosophie der Erlanger Schule basiert und die Betriebswirtschaftslehre als normative Handlungswissenschaft zu etablieren suchte (Steinmann et al. 1976; Steinmann 1978). Auf der 1982 abgehaltenen Tagung der Kommission Wissenschaftstheorie im Verband der Hochschullehrer für Betriebswirtschaft e. V. stellte man gar die programmatische Frage nach einem „Paradigmenwechsel in der Betriebswirtschaftslehre?“ (Fischer-Winkelmann 1983). Antoni (1983) kommt in seinem Beitrag zu dem Schluss, dass die ,wissenschaftstheoretische Richtung des Konstruktivismus, die die Betriebswirtschaftslehre als Kulturwissenschaft verstanden wissen will, [...] immer mehr Anhänger zu gewinnen [scheint]"; er spricht von einem sich ankündigenden „Paradigma-Switch“(S. 55).

Ein Paradigmenwechsel hin zu einem geistes- oder kulturwissenschaftlichen Forschungsideal hat zwar nicht stattgefunden, dennoch lässt sich der Betriebswirt- 
schaftslehre in der Forschungspraxis heute eine weitgehende methodologische Offenheit attestieren, die quantitative und qualitative Forschung gleichermaßen für legitim erachtet. In bestimmten Bereichen der Betriebswirtschaftslehre (z. B. Marketing, Organisationsforschung, Strategisches Management) kann die qualitative Forschung als fest etabliert gelten, und auch die letzten beiden verbliebenen deutschsprachigen wissenschaftlichen Zeitschriften der Allgemeinen Betriebswirtschaftslehre - Schmalenbachs Zeitschrift für betriebswirtschaftliche Forschung $(\mathrm{ZfbF})$ und die Betriebliche Forschung und Praxis (BFuP) - zeichnen sich durch eine ,inhaltliche und methodische Offenheit“ (ZfbF) aus bzw. vertreten einen „Methodenpluralismus, d.h. Überblicksartikel, konzeptionell-analytische, formalanalytische und empirische Arbeiten sind gleich willkommen" (BFuP).

Es stellt eine Besonderheit der deutschen Betriebswirtschaftslehre dar, dass sie im Rahmen der Allgemeinen Betriebswirtschaftslehre den Anspruch einer einheitlichen Fundierung ihrer Disziplin erhebt. Demgegenüber lässt sich beispielsweise im anglo-amerikanischen Raum eine weitgehende Zersplitterung der betriebswirtschaftlichen Teilgebiete ohne Suche nach einer einheitlichen Perspektive feststellen (Weber 2018, S. 28). In Bezug auf die geisteswissenschaftliche Öffnung der (deutschen) Betriebswirtschaftslehre wäre zu erwarten, dass die Allgemeine Betriebswirtschaftslehre bestrebt ist, den gelebten Methodenpluralismus entsprechend zu würdigen und in ihr Fundament zu integrieren. Demgegenüber finden sich jedoch immer wieder Aussagen, die eher auf das Gegenteil hinweisen, also auf das Festhalten an einer einseitig-naturwissenschaftlichen Fundierung. Pfriem (2011) konstatiert beispielsweise, ein „Blick in die aktuelle Lehrbücherwelt der (Allgemeinen) Betriebswirtschaftslehre“ würde ,,alle Vermutungen [widerlegen], von den siebziger Jahren des 20. Jahrhunderts bis heute habe ein solcher paradigmatischer Wandel tatsächlich stattgefunden“ (S. 284). Kirsch et al. (2007) kommen zu dem ähnlichen Schluss, ,dass zwischen dem tatsächlichen Spektrum dessen, was unter der Bezeichnung ,Betriebswirtschaftslehre' geforscht, publiziert und gelehrt wird, und der Programmatik dieser wissenschaftlichen Gemeinschaft eine erhebliche Diskrepanz besteht“ (S. 156f). Heise (2015) attestiert der Wirtschaftswissenschaft ,in den letzten 40 Jahren“ gar „eine paradigmatische Verengung“ (S. 377).

Ließe sich in der Allgemeinen Betriebswirtschaftslehre tatsächlich eine solche Verengung beobachten, stünde diese im Widerspruch zu der methodologischen Offenheit der Wissenschaftspraxis. Es gibt in Bezug auf eine flächendeckende Verengung der Allgemeinen Betriebswirtschaftslehre auf ein naturwissenschaftliches Forschungsideal bislang allerdings keine systematischen Untersuchungen, die dies belegen würden. Vor diesem Hintergrund besteht das Ziel des Beitrags in einem Review der wissenschaftstheoretischen Grundlagen der Allgemeinen Betriebswirtschaftslehre. Hierfür eignet sich besonders das Format des Lehrbuchs, da es einerseits lehrt, was als Standard gelten kann bzw. sich aus Sicht der Fachvertreter durchgesetzt hat, und es andererseits die Grundlage für die Sozialisation der kommenden Generation betriebswirtschaftlicher Forscher*innen darstellt und in diesem Sinne auch die Wissenschaftspraxis konstituiert. Das vorliegende Review umfasst alle seit 2005 erschienenen deutschsprachigen Lehrbücher der Allgemeinen Betriebswirtschaftslehre. 
Der Beitrag gliedert sich in drei Teile. Der erste stellt die wissenschaftstheoretische Grundlage des Reviews dar und präzisiert die Abgrenzung zwischen dem naturwissenschaftlichen Erklären und dem geisteswissenschaftlichen Verstehen. Der zweite Teil umfasst das Review. Hierbei zeigt sich, dass sich die Betriebswirtschaftslehre fast unisono als angewandte Geisteswissenschaft mit naturwissenschaftlichem Forschungsideal versteht und sich damit in der Allgemeinen Betriebswirtschaftslehre tatsächlich besagte Verengung feststellen lässt. Der dritte Teil des Beitrags stellt schließlich Überlegungen $\mathrm{zu}$ einer wissenschaftstheoretischen Revision der Allgemeinen Betriebswirtschaftslehre an, die dem praktizierten Methodenpluralismus entspricht und die praktische Relevanz verstehender Forschung aufzeigt. Damit bietet der Beitrag Anknüpfungspunkte, ,die hinter der tatsächlichen Situation herhinkende Programmatik [der Betriebswirtschaftslehre] neu zu formulieren, um sie besser mit den Gegebenheiten in Einklang zu bringen“ (Kirsch et al. 2007, S. 157).

\section{Erklären und Verstehen: eine Abgrenzung}

Historisch geht die Unterscheidung von (naturwissenschaftlichem) Erklären und (geisteswissenschaftlichem) Verstehen auf den Historiker Wilhelm Dilthey zurück, der die Geisteswissenschaft in Abgrenzung zur Naturwissenschaft als eigenständige Wissenschaft etablieren wollte. Seine Aussage „Die Natur erklären wir, das Seelenleben verstehen wir“ aus dem Jahre 1894 (Dilthey 1924, S. 144) stellt den programmatischen Ausdruck dieser bis heute einflussreichen Grundlegung der Geisteswissenschaften dar. Die Eigenständigkeit des Verstehens gegenüber dem Erklären wurde dann beispielsweise von Max Weber, Martin Heidegger, Hans-Georg Gadamer und Jürgen Habermas weiter ausgearbeitet und stellt bis heute die Grundlage der hermeneutisch vorgehenden Wissenschaften dar.

\subsection{Ontologie und Epistemologie}

Die Perspektiven des Erklärens und des Verstehens unterscheiden sich in Bezug auf die Annahmen über die Beschaffenheit des Erkenntnisgegenstands oder allgemein der Wirklichkeit (Ontologie) sowie über die Möglichkeiten der Erkenntnisgewinnung (Epistemologie). Ontologisch ist zwischen einer realistischen (auch objektivistischen) und einer konstruktivistischen (auch anti-realistischen, nominalistischen oder subjektivistischen) Sichtweise zu unterscheiden (Burrell und Morgan 1979, S. 3 f.; Scherer und Marti 2019, S. 20). Während der Realismus annimmt, dass der Erkenntnisgegenstand unabhängig vom erkennenden Subjekt existiert, geht der Konstruktivismus davon aus, dass er erst durch das erkennende Subjekt (sozial) konstruiert wird. Das Erklären beruht auf einer realistischen Sichtweise; es nimmt an, dass sich die Struktur der Wirklichkeit situations- und subjektunabhängig über Ursache-Wirkungs-Beziehungen abbilden lässt. Demgegenüber folgt das Verstehen einer konstruktivistischen Sichtweise; es geht davon aus, dass der Erkenntnisgegenstand durch den konkreten Sinnzusammenhang der agierenden Subjekte entsteht und von diesem nicht herausgelöst werden kann. Die Existenz allgemeingültiger sozialer Gesetze wird bestritten. 
Die Epistemologie beschäftigt sich mit erkenntnistheoretischen Aspekten bzw. der Möglichkeit der Wissensgewinnung. Folgt man dem Erklären, lassen sich alle Phänomene - also auch menschliche Handlungen - durch allgemeingültige Ursache-Wirkungs-Beziehungen abbilden. Theorien sind axiomatisch fundierte Aussagensysteme über die Struktur kausal-determinierter Ablaufgesetze, deren Kenntnis eine Prognose des zu erklärenden Phänomens erlaubt, also Aussagen darüber zu treffen, unter welchen Bedingungen bestimmte Ereignisse auftreten (Wenn-DannAussagen). Der theoretische Erklärungs- bzw. Prognosegehalt bemisst sich darin, inwiefern die Theorie mit empirisch vorgefundenen Sachverhalten übereinstimmt (Kirsch et al. 2007, S. 7 ff.). Die empirische Prüfung einer Theorie ist grundsätzlich auf induktivem oder deduktivem Wege möglich. Bei der induktiven Generierung einer Theorie werden aus empirisch feststellbaren Besonderheiten allgemeingültige Gesetze abgeleitet. Bei der deduktiven Überprüfung einer Theorie wird die Allgemeingültigkeit von Theorien dagegen durch empirisch feststellbare Besonderheiten verifiziert oder falsifiziert (Pepels 2017, S. 4f.). In beiden Fällen nimmt der/die Wissenschaftler*in eine passive Beobachterrolle ein, d.h., das Untersuchungsobjekt wird aus der Außenperspektive betrachtet.

Demgegenüber findet beim Verstehen ein Wechsel von der Außen- in die Binnenperspektive statt. Der/die Wissenschaftler*in taucht in eine aktive Beobachterrolle ein und nimmt an den untersuchten sozialen Interaktionen teil (Kirsch 1997, S. 73; Sandberg und Tsoukas 2011, S. 340ff.). Wissenschaftliche Erkenntnis stellt eine (soziale) Konstruktion dar, die nur in einem bestimmten und durch den Beobachter mitgestalteten Sinnzusammenhang Gültigkeit besitzt. Die Epistemologie des Verstehens ist die Hermeneutik, die auch als Lehre des Verstehens oder des interpretativen Vorgehens bezeichnet wird (Kromphardt et al. 1979, S. 80; Soeffner 2017, S. 164). Das Ziel hermeneutischer Bestrebungen besteht in der Erschließung von Sinn- und Wirkungszusammenhängen (Kornmeier 2007, S. 80). Sinnzusammenhänge beziehen sich auf Bedeutungsstrukturen, die Subjekte ihren Handlungen und ihrer Umwelt unterlegen (Kieser 1995, S. 7). Bei den Wirkungszusammenhängen geht es zwar wie beim Erklären um Ursache-Wirkungs-Beziehungen, im Vordergrund stehen dabei aber keine linearen, sondern zirkuläre Kausalitäten (Julmi 2019, S. 245 ff.; Schultze und Stabell 2004, S. 554), die sich auf die wechselseitigen Abhängigkeiten realer Gegebenheiten beziehen. Auf theoretischer Ebene drücken sich zirkuläre Kausalitäten beispielsweise in bidirektionalen Beziehungen, rekursiven Mustern oder gegenseitig aufeinander bezogenen Interaktionen aus (Sandberg und Tsoukas 2011, S. 352). Erkenntnistheoretisch findet sich die zirkuläre Kausalität im hermeneutischen Zirkel wider, nach dem sich einzelne Sachverhalte immer nur vor ihrem Hintergrund als Kontext erschließen lassen, während auf der anderen Seite der Kontext nur über die Betrachtung einzelner Sachverhalte erschlossen werden kann, so dass zwischen Einzelnem und Ganzem ein wechselseitiges Verhältnis besteht (Kornmeier 2007, S. 80; Weik 2005, S. 99).

\subsection{Methodologie und Methode}

Die Methodologie trifft Aussagen über die generelle Logik und das Vorgehen bei der Generierung von (wissenschaftlichem) Wissen. Sie beinhaltet ontologische und 
epistemologische Annahmen und impliziert ein generelles Forschungsdesign. Die Methode bezieht sich dagegen ausschließlich auf spezifische Strategien und Techniken bezüglich der Gewinnung, Analyse, Interpretation, Validität und Reliabilität von Daten bzw. Informationen (Bogdan und Biklen 2007). Die Methodologie ist daher breiter und abstrakter gefasst als die Methode; sie unterstützt Forschende bei der Auswahl und Bewertung geeigneter Methoden und ist wissenschaftstheoretisch fundiert.

Auf methodologischer Ebene besteht ein prinzipieller Unterschied zwischen der quantitativen und der qualitativen Methodologie (Cohen et al. 2018; Creswell und Creswell 2018). Die quantitative Methodologie folgt der Ontologie und der Epistemologie des Erklärens und stellt eine Reihe von Methoden zur Erforschung des Erkenntnisgegenstands zur Verfügung (z.B. Regressionsanalyse, Strukturgleichungsmodelle). Die qualitative Forschung folgt dagegen der Ontologie und Epistemologie des Verstehens und umfasst ebenfalls ein umfassendes Methodenrepertoire, das sich bei der Forschung einsetzen lässt (z. B. Dokumentenanalyse, teilnehmende Beobachtung, Sequenzanalyse). Alternativ wird zwischen der szientistischen Methodologie (des Erklärens) und der hermeneutischen Methodologie (des Verstehens) (Hitzler und Eberle 2017, S. 118) oder, auf Wilhelm Windelband zurückgehend, zwischen nomothetisch-naturwissenschaftlicher und idiographisch-geisteswissenschaftlicher Forschung unterschieden (Groß 2019).

Die Differenzierung von Methodologie und Methode ist für eine wissenschaftstheoretische Diskussion natur- und geisteswissenschaftlicher Forschung unabdingbar, da sich eine gegenseitige Ausschließlichkeit oder Inkommensurabilität (Scherer 1998) nur auf methodologischer (sowie auf ontologischer und epistemologischer), aber nicht auf methodischer Ebene ergibt. Eine wissenschaftliche Arbeit kann nicht gleichzeitig einer erklärenden und einer verstehenden Methodologie folgen, wohl aber erklärende und verstehende Methoden einsetzen. Wenn Pietsch anführt, ,eine strenge Unterscheidung zwischen Erklären und Verstehen“ werde ,mittlerweile kritisch gesehen“ (2015, S. 122), kann dies nur auf methodischer Ebene geltend gemacht werden. Hier gilt es als anerkannt, dass auch das Erklären eines vorgängigen Verstehens bedarf und in diesem Sinne auf hermeneutischen Verfahren beruht oder zumindest beruhen kann (Kirsch et al. 2007, S. 72; Strübing 2018, S. 6). Dies gilt analog für die Interpretation von Ergebnissen, deren Relevanz sich nur vor dem Hintergrund eines Sinnzusammenhangs aufzeigen lässt, oder wenn qualitative Methoden im Zuge der Datenbeschaffung genutzt, letztlich aber numerisch übersetzt und quantitativ verarbeitet werden. Auf der anderen Seite wird mitunter auch in der qualitativen Forschung auf quantitative Daten zurückgegriffen, so dass auch im Rahmen des Verstehens erklärende Methoden zum Einsatz kommen (können).

In Bezug darauf, was die Geisteswissenschaften (und damit auch die Betriebswirtschaftslehre) auf methodologischer Ebene für zulässig erachtet, gilt: Wird alleine die Methodologie des Erklärens für legitim erachtet, stellt dies einen Methodenmonismus zwischen Natur- und Geisteswissenschaften dar - eine Haltung, die ihren programmatischen Ausdruck in der Einheit der Wissenschaft findet. Wer umgekehrt eine strikte Trennung zwischen der Methodologie der Natur- und Geisteswissenschaften fordert und für Letztere alleine die Methodologie des Verstehens zulässt, vertritt einen Methodendualismus (Kieser 1995, S. 6ff.; Strübing 2018, S. 5). Als 
dritter Weg sieht der Methodenpluralismus quantitative und qualitative Methodologien als einander ergänzend an, die jeweils auf ihre Weise zu einem umfassenden und facettenreichen Bild in Bezug auf einen Forschungsgegenstand oder eine Disziplin beitragen. Die im nachfolgenden Review zu klärende Frage besteht diesbezüglich darin, ob sich die Betriebswirtschaftslehre in ihrem Selbstverständnis zu einer methodenpluralistischen Disziplin gewandelt hat oder nach wie vor einen Methodenmonismus vertritt.

\section{Durchführung und Ergebnisse des Reviews}

Das nachfolgende Review arbeitet nach der Darstellung der Literatursuche und -auswahl zunächst das Selbstverständnis der Betriebswirtschaftslehre auf. Im Vordergrund stehen hierbei drei Fragen: Inwiefern sieht sich die deutsche Betriebswirtschaftslehre als abgrenzbaren Forschungskreis? Wo ordnet sie sich im System der Wissenschaften ein? Inwiefern versteht sie sich als anwendungsorientierte Disziplin? Von der Beantwortung dieser Fragen ausgehend erörtert der Beitrag mit besonderem Augenmerk auf dem Verstehen bzw. der Hermeneutik, welche Prozesse und Möglichkeiten der Erkenntnisgewinnung im Rahmen der Allgemeinen Betriebswirtschaftslehre für zulässig erachtet werden.

\subsection{Literatursuche und -auswahl}

Für die Literaturrecherche wurde die öffentlich zugängliche Datenbank der Deutschen Nationalbibliothek (DNB) herangezogen (Deutsche Nationalbibliothek 2019). Die DNB ist die zentrale Archivbibliothek für alle Bücher in deutscher Sprache aus dem In- und Ausland. Sie umfasst damit auch alle in einem ordentlichen Verlag veröffentlichten deutschsprachigen Lehrbücher der ABWL. Das Review berücksichtigt alle seit 2005 erschienenen Lehrbücher, wobei bei mehreren Auflagen nur die neueste einbezogen wurde. Wir führten die Recherche am 28.10.2019 mit insgesamt zwei Suchanfragen durch. Die erste Suchanfrage beinhaltete die Suchkriterien ,Allgemeine“ und „Betriebswirtschaftslehre“ und führte zu 5262 Treffern bzw. 1830 Treffern der Jahre seit 2005. Die zweite Suchanfrage basierte auf den Suchkriterien ,Einführung“ und „Betriebswirtschaftslehre“ und resultierte in 1243 Treffern bzw. 517 Treffern seit 2005.

Die entstandene Liste von 2347 Büchern, die aufgrund der Ähnlichkeit beider Suchanfragen zahlreiche Dubletten enthielt, durchsuchten wir manuell nach Lehrbüchern der ABWL. Es wurden nur Lehrbücher berücksichtigt, die seit 2005 als Erstauflage oder in einer überarbeiteten Neuauflage erschienen sind und im Kern explizit die Betriebswirtschaftslehre behandeln. Nachdrucke älterer Lehrbücher fanden ebenso wenig Berücksichtigung wie Lehrbücher über Wirtschaftswissenschaften oder Managementlehre. Ebenfalls nicht berücksichtigt wurden Lehrbücher, die sich an ein spezifisches Publikum richten. Hierunter fielen etwa Lehrbücher für Ingenieure, für Studierende der Geografie und Ernährungswissenschaften, für Betriebsräte oder von Frau zu Frau. Analoges galt für Lexika, Arbeitsbücher, Aufgabenhefte, Polemiken oder Herausgeberbände. Des Weiteren fanden Lehrbücher keine Be- 


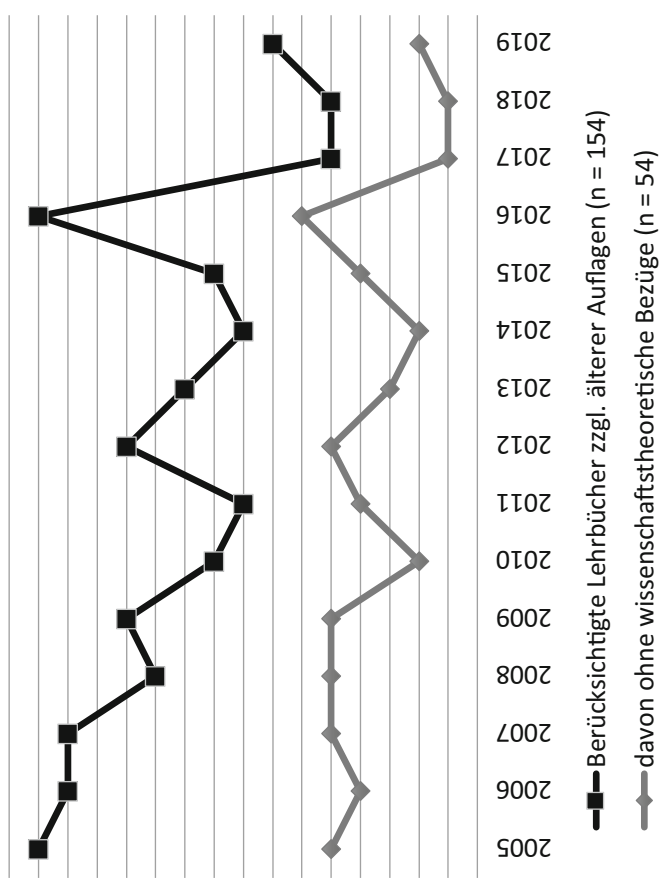

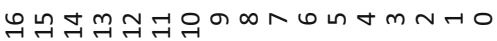

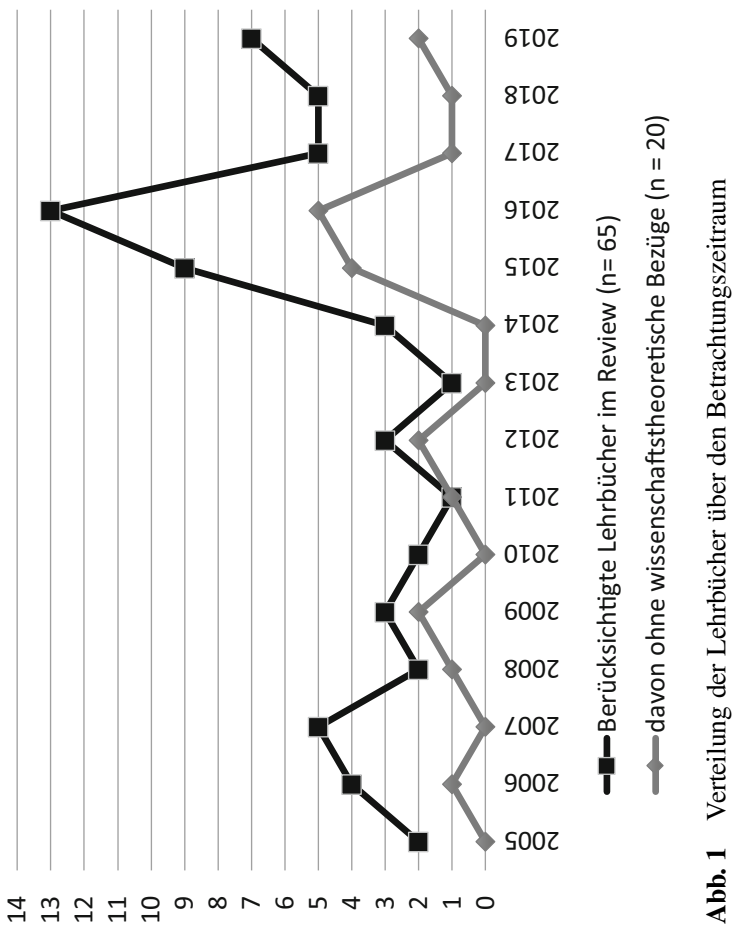


rücksichtigung, die eine spezifische theoretische Perspektive einnehmen (z.B. aus institutionenökonomischer Sicht) oder auf ein Thema fokussieren (z. B. auf die Geschichte der Betriebswirtschaftslehre). Verlagsseitig wurden Bücher ausgeschlossen, deren Veröffentlichung auf einem Self-Publishing-Format beruht. Das Lehrbuch von Pepels (2014) haben wir ausgeschlossen, da mit Pepels (2017) ein neueres und umfangreicheres Werk desselben Autors existiert, das die wissenschaftstheoretischen Überlegungen des früheren Werks spiegelt. Ebenfalls nicht berücksichtigt haben wir das Buch von Pfriem (2011), das eher eine (wenngleich erhellende und weiter unten aufgegriffene) Kritik an der Betriebswirtschaftslehre als ein Lehrbuch der Allgemeinen Betriebswirtschaftslehre darstellt. Das Lehrbuch von Brockhoff (2017) fand keine Berücksichtigung, da es sich um eine historische Betrachtung handelt, die ,etwa in den 1970er Jahren“ endet (S. 253). Demgegenüber wurden vier Lehrbücher mit in die Liste aufgenommen, die zwar keine Lehrbücher der Allgemeinen Betriebswirtschaftslehre im engeren Sinne darstellen, sich aber schwerpunktmäßig mit deren wissenschaftstheoretischen Grundlagen auseinandersetzen und damit für die vorliegende Untersuchung aufschlussreich sind. Dies betrifft die Lehrbücher von Eisend und Kuß (2017), Helfrich (2016), Kirsch et al. (2007) sowie Kornmeier (2007). Die manuelle Prüfung konnte die Liste auf insgesamt 65 Titel reduzieren.

Die Allgemeine Betriebswirtschaftslehre befasst sich mit den „Fundamenten und den tragenden Teilen des Lehr- und Forschungsgebäudes Betriebswirtschaftslehre“ (Bardmann 2019, S. 6) und damit in ihrem Kern (auch) mit ihren wissenschaftstheoretischen Grundannahmen. Dennoch kommen 20 der 65 berücksichtigten Lehrbücher gänzlich ohne wissenschaftstheoretische Verweise aus. Hierbei handelt es sich um die Lehrbücher von Amely und Krickhahn (2016), Arnold (2016), Geyer und Ahrendt (2016), Harbrücker (2008), Hutzschenreuter (2015), Klockhaus (2012), Kreuzer (2019), Kuhlmann und Sauter (2009), Lamprea (2009), Mentzel (2015), Messner et al. (2016), Mihalic (2012), Paul (2015), Pilz (2017), Schäffer (2015), Schultz (2016), Teuscher (2011), Vettiger (2006), Voss (2018) und Wettengl (2018). Diese finden in der nachfolgenden Analyse entsprechend keine Berücksichtigung. In den übrigen 45 Lehrbüchern finden sich wissenschaftstheoretische Bezüge, wobei das Spektrum von rudimentären Angaben bis zu umfassenden Auseinandersetzungen reicht.

Abb. 1 zeigt die Anzahl der berücksichtigten Lehrbücher je Erscheinungsjahr im Betrachtungszeitraum. Die untere Linie spiegelt jeweils den Anteil an Lehrbüchern ohne wissenschaftstheoretische Bezüge wider. Während das linke Diagramm die im Review berücksichtigten Lehrbücher zeigt, sind im rechten Diagramm zusätzlich ältere Auflagen mit einbezogen. Dadurch lässt sich nachzeichnen, wie viele Lehrbücher der Allgemeinen Betriebswirtschaftslehre in den jeweiligen Jahren erschienen sind. Ein leichter Abwärtstrend ist nicht zu leugnen.

Epistemologische bzw. methodologische Aussagen finden sich in 38 Lehrbüchern, so dass sieben Lehrbücher zwar wissenschaftstheoretische Aussagen enthalten, aber keine, die für diese Untersuchung relevant sind. Dies sind die Lehrbücher von Bernecker (2009), Kußmaul (2016), Mülder und Lorberg (2015), Schulte (2007), Straub (2015), Wiltinger und Wiltinger (2005) sowie Wöhe et al. (2016). Einige davon enthalten aber aufschlussreiche Aussagen für die nachfolgende Darlegung des Selbstverständnisses der Betriebswirtschaftslehre. 


\subsection{Das Selbstverständnis der Betriebswirtschaftslehre}

Die Ausführungen in den Lehrbüchern zur Geschichte der Betriebswirtschaftslehre zeigen, dass sich die deutschsprachige Betriebswirtschaftslehre (nach wie vor) als eigenen, von der internationalen Managementforschung abzugrenzenden Forschungskreis ansieht. In Bezug auf die Entwicklung ab dem Ende des 19. Jahrhunderts wird fast ausschließlich auf deutsche Denker wie Eugen Schmalenbach (1873-1955), Heinrich Nicklisch (1876-1946), Wilhelm Rieger (1878-1971), Erich Gutenberg (1897-1984) oder Edmund Heinen (1919-1996) rekurriert. Die in Österreich erschienenen Bücher von Egger et al. (2016) und Schauer (2019) gehen ergänzend auf den österreichischen Beitrag zur (deutschsprachigen) Betriebswirtschaftslehre ein, ohne deshalb eine andere Entwicklungslinie aufzuzeigen. Schweitzer und Baumeister (2015) führen an, dass der Wissenschaftsbetrieb heute zwar international sei, die „deutschsprachige Betriebswirtschaftslehre“ aufgrund der recht späten Internationalisierung jedoch ,Sonderwege beschreiten [konnte]“ (S. 61).

In 31 der 65 Lehrbücher wird eine (Selbst-)Verortung der Betriebswirtschaftslehre im System der Wissenschaften vorgenommen. Die Betriebswirtschaftslehre wird wahlweise den Geisteswissenschaften $(12 \times)$, den Kulturwissenschaften $(8 \times)$, den Sozialwissenschaften $(8 \times)$, den Geistes- und Kulturwissenschaften $(1 \times)$, den Geistes- und Sozialwissenschaften $(1 \times)$ oder den Geistes-, Kultur- und Sozialwissenschaften $(1 \times)$ zugerechnet, wobei diese jeweils das Pendant zu den Naturwissenschaften darstellen. Es erscheint daher angemessen, die Betriebswirtschaftslehre als Geisteswissenschaft zu bezeichnen und Kultur- bzw. Sozialwissenschaften als Synonyme zu betrachten. Darüber hinaus fällt der heterogene Gebrauch des Begriffs der Sozialwissenschaften auf. Neben der Verwendung des Begriffs als Pendant zu den Naturwissenschaften werden die Sozialwissenschaften verortet

- als Teilbereich der Geistes- oder Kulturwissenschaften (Beschorner und Peemöller 2006; Birker 2006; Kocian-Dirr 2019; Kußmaul 2016; Lange 2018; Schneck 2010; Vahs und Schäfer-Kunz 2015; Weber et al. 2018; Wiltinger und Wiltinger 2005),

- neben den Geisteswissenschaften als Teilbereich der Kulturwissenschaften (Deelmann 2015),

- neben den Kulturwissenschaften als Teilbereich der Geisteswissenschaften (Pepels 2017),

- neben den Geisteswissenschaften und neben den Naturwissenschaften (Erdmann und Krupp 2018) sowie

- neben den Wirtschaftswissenschaften als Teilbereich der Geisteswissenschaften (Helfrich 2016; Mentzel 2013; Mülder und Lorberg 2015).

Sieht man von dem offenbar interpretationswürdigen Begriff der Sozialwissenschaften $a b$, ergibt sich insgesamt eine recht einheitliche Verortung der Betriebswirtschaftslehre im System der Wissenschaften. Abb. 2 zeigt diesen ,Konsens“ im Überblick. Statt Formal- ließe sich auch Ideal-, statt Geistes- auch Kulturwissenschaften einsetzen.

Für die weitere Analyse aufschlussreich ist zudem die Frage, inwiefern es sich bei der Betriebswirtschaftslehre um eine reine oder eine angewandte Wissenschaft handelt. Für die Vertreter der reinen Wissenschaft ist das Ziel der Wissenschaft 


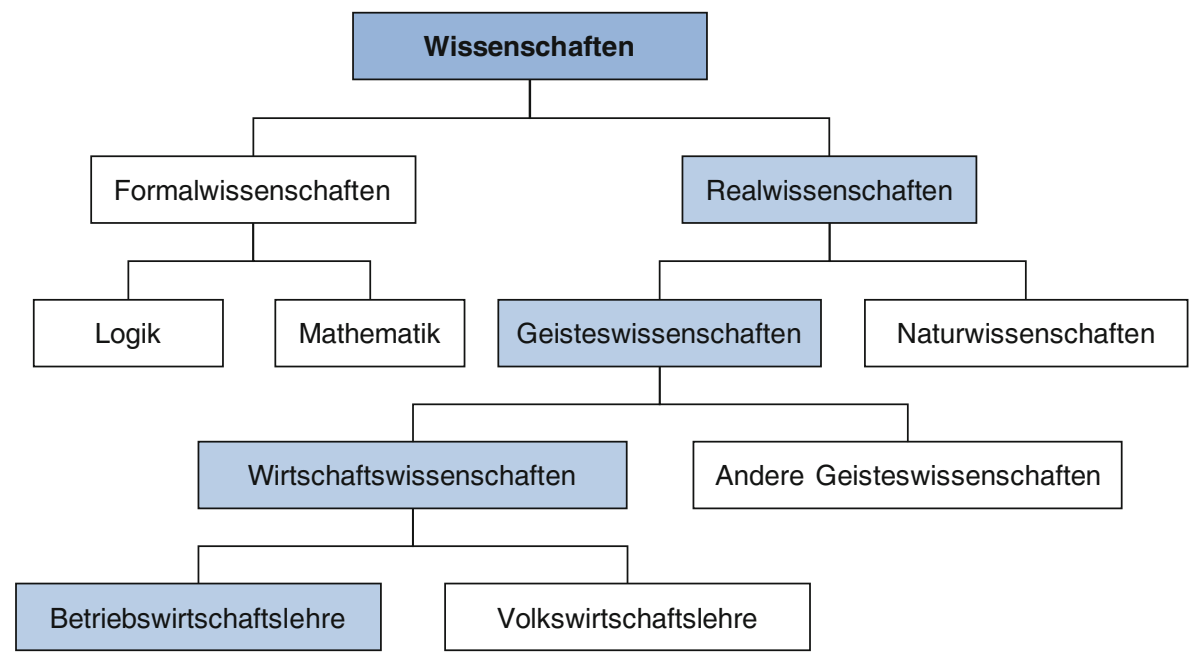

Abb. 2 Die Betriebswirtschaftslehre im System der Wissenschaften

alleine der Erkenntnisfortschritt, während Anhänger der angewandten Wissenschaft das Ziel verfolgen, die gewonnenen Erkenntnisse praktisch umzusetzen und so dem Menschen zu einer besseren Lebensbewältigung zu verhelfen (Kornmeier 2007, S. 22).

In 37 der berücksichtigten Bücher finden sich Aussagen zur diesbezüglichen Charakterisierung der Betriebswirtschaftslehre. Hierbei zeigt sich eine klare Tendenz in Richtung Anwendungsorientierung. Lediglich bei Egger et al. (2016) finden sich ausschließlich Aussagen zu einer theoretischen Zielsetzung der Betriebswirtschaftslehre (S. 31). Alle übrigen Autoren betonen deren praktisches Ziel bzw. deren praktische Aufgabe, wobei sich in Bezug auf das Verhältnis des theoretischen und des praktischen Teils grob zwei Gruppen differenzieren lassen.

Die erste, sich in der Mehrzahl befindende Gruppe sieht die theoretische und die praktische Zielsetzung als gleichwertig an. Dies wird besonders bei Jung (2016) deutlich:

Beide Teile unterscheiden sich in ihrem Erkenntnisziel. Die theoretische Betriebswirtschaftslehre dient ausschließlich der Wissenserlangung, unabhängig vom Grad der praktischen Anwendbarkeit, während die angewandte Betriebswirtschaftslehre immer eine praxisorientierte Forschung betreibt und ihre Aufgabe in der Beschreibung und Beurteilung von empirisch vorgefundenen Entscheidungsprozessen sowie in der Entwicklung neuer Entscheidungsgrundlagen sieht. (S. 24)

Demgegenüber sieht die zweite Gruppe die Betriebswirtschaftslehre primär als angewandte Wissenschaft, deren praktische Aussagen auf theoretischen Aussagensystemen beruhen. Die Entwicklung von Theorien wird nicht als eigenständiges Ziel, sondern als Mittel zur Erreichung des praktischen Ziels angesehen. Der wesentliche Unterschied zur ersten Gruppe besteht also darin, dass die theoretische Forschung 
Abb. 3 Die Zielsetzung(en) der Betriebswirtschaftslehre

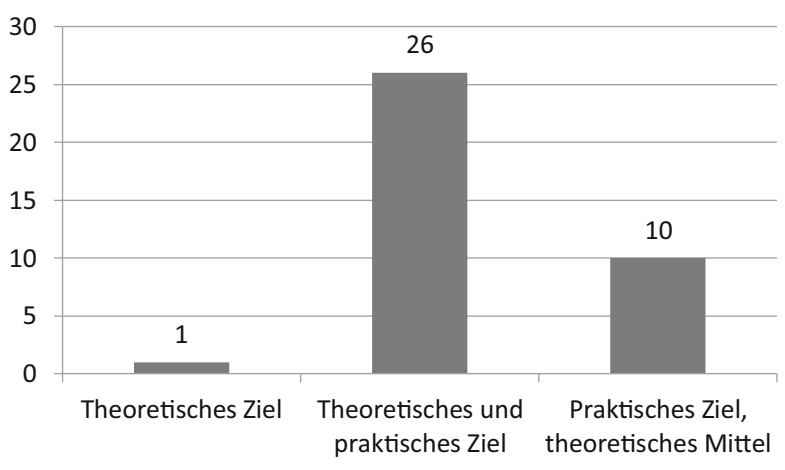

nicht von der Lösung praktischer Probleme als ihr Hintergrund bzw. ihre Legitimationsgrundlage getrennt werden kann. Aus dieser Sicht umfasst anwendungsorientierte Wissenschaft ,solche Tätigkeiten, die im Wesentlichen darauf ausgerichtet sind, mithilfe von Erkenntnissen der theoretischen oder Grundlagenwissenschaften sowie der Erfahrung der Praxis Problemlösungen (Regeln, Modelle, Verfahren) für praktisches Handeln zu entwickeln“ (Thommen et al. 2017, S. 16f.).

Abb. 3 zeigt die Verteilung der Ansichten über die Zielsetzung(en) der Betriebswirtschaftslehre im Überblick. Über beide Gruppen hinweg wird deutlich, dass sich die Betriebswirtschaftslehre heute (fast) unisono als anwendungsorientierte Wissenschaft versteht.

\subsection{Prozess und Möglichkeiten der Erkenntnisgewinnung}

Zur Beantwortung der Frage, ob sich die Betriebswirtschaftslehre in ihrem Selbstverständnis von einer methodenmonistischen zu einer methodenpluralistischen Disziplin gewandelt hat, sind epistemologische bzw. methodologische Aussagen in den Lehrbüchern zu untersuchen. Hierzu finden sich in 38 Lehrbüchern Aussagen. Tab. 1 zeigt, welche Erkenntnisziele die Autoren jeweils für die Betriebswirtschaftslehre geltend machen und auf welchem Wege diese erreicht werden. Für das Lehrbuch von Olfert und Rahn (2017) haben wir die Erkenntnisziele des Erklärens und Gestaltens ergänzt, obwohl diese nicht explizit genannt werden. Die Autoren verweisen auf die Möglichkeiten der Induktion und Deduktion (S. 28) und damit implizit auf das naturwissenschaftliche Erklären. Da sie zudem eine theoretische und eine praktische Ausrichtung der Betriebswirtschaftslehre differenzieren (S. 25), kann von dem praktischen Erkenntnisziel des Gestaltens ausgegangen werden.

In Bezug auf die Erkenntnisziele ergibt sich ein eindeutiges Bild. Alle 38 Autoren sehen die Erklärung - also die Erfassung kausaler Wirkungszusammenhänge in der Form von Wenn-Dann-Aussagen - als theoretisches Erkenntnisziel der Betriebswirtschaftslehre, während das Verstehen in lediglich 2 von 38 Lehrbüchern überhaupt Erwähnung findet. Außer bei Egger et al. (2016), die der Betriebswirtschaftslehre nur ein theoretisches Erkenntnisziel zuschreiben, sehen zudem alle Autoren die Gestaltung als praktisches Ziel an, wobei die Verbindung zwischen der theoretischen und der praktischen Zielsetzung meist über die Prognose der erklärten Sachverhalte 
Tab. 1 Erkenntnisziele und Möglichkeiten der Erkenntnisgewinnng

\begin{tabular}{|c|c|c|c|c|c|c|}
\hline \multirow{3}{*}{$\frac{\text { Quelle }}{\text { Backhaus et al. (2010) }}$} & \multicolumn{3}{|c|}{ Erkenntnisziele } & \multicolumn{3}{|c|}{ Erkenntnisgewinnung } \\
\hline & \multicolumn{2}{|c|}{ Theoretisch } & \multirow{2}{*}{$\frac{\text { Praktisch }}{\text { Gestalten }}$} & \multicolumn{2}{|l|}{$\mathrm{NaWi}$} & \multirow{2}{*}{$\frac{\mathrm{GeWi}}{-}$} \\
\hline & Erklären & - & & - & - & \\
\hline $\begin{array}{l}\text { Balderjahn und Specht } \\
\text { (2016) }\end{array}$ & Erklären & - & Gestalten & Induktion & Deduktion & Hermeneutik \\
\hline Bardmann (2019) & Erklären & - & Gestalten & Induktion & Deduktion & - \\
\hline $\begin{array}{l}\text { Beschorner und } \\
\text { Peemöller (2006) }\end{array}$ & Erklären & - & Gestalten & Induktion & Deduktion & Hermeneutik \\
\hline Birker (2006) & Erklären & - & Gestalten & Induktion & Deduktion & - \\
\hline $\begin{array}{l}\text { Corsten und Corsten } \\
(2019)\end{array}$ & Erklären & - & Gestalten & Induktion & Deduktion & Hermeneutik \\
\hline Deelmann (2015) & Erklären & - & Gestalten & - & - & - \\
\hline $\begin{array}{l}\text { Domschke und Scholl } \\
\text { (2008) }\end{array}$ & Erklären & - & Gestalten & - & - & - \\
\hline Egger et al. (2016) & Erklären & - & - & Induktion & Deduktion & Hermeneutik \\
\hline $\begin{array}{l}\text { Eichhorn und Merk } \\
\text { (2016) }\end{array}$ & Erklären & - & Gestalten & Induktion & Deduktion & - \\
\hline $\begin{array}{l}\text { Eisend und Kuß } \\
\text { (2017) }\end{array}$ & Erklären & - & Gestalten & Induktion & Deduktion & - \\
\hline $\begin{array}{l}\text { Erdmann und Krupp } \\
\text { (2018) }\end{array}$ & Erklären & - & Gestalten & - & - & - \\
\hline Hammer (2012) & Erklären & - & Gestalten & Induktion & Deduktion & Hermeneutik \\
\hline Helfrich (2016) & Erklären & - & Gestalten & Induktion & Deduktion & Hermeneutik \\
\hline Hummel (2007) & Erklären & - & Gestalten & Induktion & Deduktion & - \\
\hline Jung (2016) & Erklären & - & Gestalten & Induktion & Deduktion & Hermeneutik \\
\hline Känel (2018) & Erklären & - & Gestalten & - & - & - \\
\hline Kirsch et al. (2007) & Erklären & Verstehen & Gestalten & - & Deduktion & Hermeneutik \\
\hline Kocian-Dirr (2019) & Erklären & - & Gestalten & - & - & - \\
\hline Kornmeier (2007) & Erklären & - & Gestalten & Induktion & Deduktion & - \\
\hline Lange (2018) & Erklären & - & Gestalten & Induktion & Deduktion & - \\
\hline Mentzel (2013) & Erklären & - & Gestalten & - & - & - \\
\hline $\begin{array}{l}\text { Olfert und Rahn } \\
\text { (2017) }\end{array}$ & [Erklären] & - & [Gestalten] & Induktion & Deduktion & - \\
\hline $\begin{array}{l}\text { Opresnik und Renn- } \\
\text { hak (2015) }\end{array}$ & Erklären & - & Gestalten & - & - & - \\
\hline Pepels (2017) & Erklären & - & Gestalten & Induktion & Deduktion & - \\
\hline Peters et al. (2005) & Erklären & - & Gestalten & Induktion & Deduktion & - \\
\hline Schauer (2019) & Erklären & - & Gestalten & Induktion & Deduktion & - \\
\hline $\begin{array}{l}\text { Schierenbeck und } \\
\text { Wöhle (2016) }\end{array}$ & Erklären & - & Gestalten & - & - & - \\
\hline $\begin{array}{l}\text { Schmalen und Pechtl } \\
\text { (2013) }\end{array}$ & Erklären & - & Gestalten & - & - & - \\
\hline Schneck (2010) & Erklären & Verstehen & Gestalten & Induktion & Deduktion & Hermeneutik \\
\hline $\begin{array}{l}\text { Schweitzer und Bau- } \\
\text { meister (2015) }\end{array}$ & Erklären & - & Gestalten & - & - & - \\
\hline Sigloch et al. (2015) & Erklären & - & Gestalten & - & - & - \\
\hline
\end{tabular}


Tab. 1 (Fortsetzung)

\begin{tabular}{|c|c|c|c|c|c|c|}
\hline \multirow{3}{*}{$\frac{\text { Quelle }}{\text { Sturm (2006) }}$} & \multicolumn{3}{|c|}{ Erkenntnisziele } & \multicolumn{3}{|c|}{ Erkenntnisgewinnung } \\
\hline & \multicolumn{2}{|c|}{ Theoretisch } & \multirow{2}{*}{$\frac{\text { Praktisch }}{\text { Gestalten }}$} & \multicolumn{2}{|l|}{$\mathrm{NaWi}$} & \multirow{2}{*}{$\frac{\mathrm{GeWi}}{-}$} \\
\hline & Erklären & - & & Induktion & Deduktion & \\
\hline $\begin{array}{l}\text { Thommen et al. } \\
\text { (2017) }\end{array}$ & Erklären & - & Gestalten & - & - & - \\
\hline Töpfer (2007) & Erklären & - & Gestalten & - & Deduktion & - \\
\hline $\begin{array}{l}\text { Vahs und Schäfer- } \\
\text { Kunz (2015) }\end{array}$ & Erklären & - & Gestalten & - & - & - \\
\hline Weber et al. (2018) & Erklären & - & Gestalten & Induktion & Deduktion & - \\
\hline Wehrlin (2014) & Erklären & - & Gestalten & Induktion & Deduktion & Hermeneutil \\
\hline Gesamt & $38 / 38$ & $2 / 38$ & $37 / 38$ & $22 / 38$ & $24 / 38$ & $10 / 38$ \\
\hline
\end{tabular}

NaWi Naturwissenschaften, GeWi Geisteswissenschaften

gesehen wird. Die Erklärung eines Sachverhalts ,,bedeutet, ihn aus Hypothesen über Gesetzmäßigkeiten und gewissen Randbedingungen logisch abzuleiten“ (Bardmann 2019, S. 83). Die Erklärung erlaubt dann schließlich die Prognose eines Sachverhalts oder Ereignisses. Die Prognose ist zwar noch auf theoretischer Ebene zu verorten (Egger et al. 2016, S. 31), stellt aber das Bindeglied für die Gestaltung auf praktischer Ebene, auf der Aussagen darüber getroffen werden, ,welche Maßnahmen geeignet sind, um bestimmte Ziele zu erreichen“ (Weber et al. 2018, S. 29). Dieser Prozess, theoretische Wenn-Dann- in praktische Um-Zu-Aussagen zu transformieren, wird als „technologische Aufgabe“ (Corsten und Corsten 2019, S. 10) oder ,technologische Funktion“ (Weber et al. 2018, S. 29) der Betriebswirtschaftslehre bezeichnet. Eine Technologie ist ein praktisches Aussagensystem, das ,die theoretischen Ursachen-Wirkungsaussagen instrumental umformt und in ihr wissenschaftliches ZielMittel-System einbaut“" (Schierenbeck und Wöhle 2016, S. 8).

Die einzigen Lehrbücher, die das Verstehen als Alternative zum Erklären behandeln, sind diejenigen von Kirsch et al. (2007) und Schneck (2010). Erstere verstehen dies explizit als einen der wesentlichen Beiträge und Besonderheiten ihrer wissenschaftstheoretischen Einführung in die Betriebswirtschaftslehre:

Während die meisten Bücher zur Wissenschaftstheorie - auch im Bereich der Betriebswirtschaftslehre - an dem Vorgehen der Naturwissenschaften orientiert sind, arbeiten wir die Besonderheiten sozialwissenschaftlicher Forschung heraus. (Kirsch et al. 2007, S. 1)

Schneck (2010) sieht das Verstehen ebenfalls als relevantes Erkenntnisziel für die Betriebswirtschaftslehre an und führt es im Zusammenhang mit der Hermeneutik als „die Lehre vom nachfühlenden Verstehen“ ein. Allerdings sei es strittig, „ob diese Methode der Erfassung von Sinnzusammenhängen im Gegensatz zur Erklärung von Wirkungszusammenhängen für die Betriebswirtschaftslehre geeignet ist". Sie werde ,jedenfalls selten in der Wirtschaftswissenschaft angewandt“ (Schneck 2010, S. 264). Der Autor sieht das Verstehen zudem als Voraussetzung einer (adäquaten) Gestaltung, denn nur ,wer den zu betrachtenden Gegenstand von vielen Blickwinkeln betrachtet, kann letztlich verstehen und dann gestalten." (Schneck 2010, S. 246). 
In Bezug auf die Möglichkeiten der Erkenntnisgewinnung werden in den meisten Lehrbüchern, die hierzu Aussagen treffen, nur Induktion und Deduktion für zulässig erachtet. In lediglich 10 der 38 Lehrbücher finden sich (eher sparsame) Hinweise zur Hermeneutik als zulässige Methode der Betriebswirtschaftslehre. Balderjahn und Specht (2016) führen neben der Induktion und der Deduktion als drittes Erkenntnisprinzip die Hermeneutik an: „Hier dient der menschliche Verstand als Erkenntnisquelle. Es ist eine Methode zur gedanklichen Auslegung (Interpretation) und Durchdringung von Problemen (Problemlösung, analytische Methode)“ (S. 27). Weitere Hinweise finden sich nicht. Auf der anderen Seite schreiben die Autoren, die Betriebswirtschaftslehre ziele ,,auf die Beschreibung, Erklärung, Prognose und Gestaltung“ einzelwirtschaftlicher Phänomene (S. 21). Die Hermeneutik kann demzufolge höchstens in einem vorwissenschaftlichen Sinne (also methodisch) zum Einsatz kommen. Das naturwissenschaftliche Ideal bleibt ebenso unberührt wie die methodenmonistische Grundlegung des Buches. Dies lässt sich analog für die Ausführungen von Egger et al. (2016) konstatieren, die die Hermeneutik neben Induktion und Deduktion als allgemeine Grundlagenmethode zulassen, wobei die Autoren ihre Sicht auf das Verstehen als Vorwissenschaft explizit machen: „Das Verstehen beschreibender (deskriptiver) Sachverhalte vermag erklärende (explikative) Untersuchungen anzuregen und zu steuern“ (S. 45). Jung (2016) beschreibt die Hermeneutik als verstehende Methode, die ,meist zur Gewinnung von Erkenntniszielen und Hypothesen“ (S. 41) und damit vorwissenschaftlich Anwendung findet. Wehrlin (2014) listet die Hermeneutik als wissenschaftliche Methode zur ,Vorselektion von Erklärungshypothesen“ (S. 10) auf; die beiden Zielsetzungen der Betriebswirtschaftslehre bleiben „eine Erklärungs- und eine Gestaltungsaufgabe“ (S. 7) und sind damit naturwissenschaftlich fundiert. Ähnlich sehen Beschorner und Peemöller (2006) den Nutzen der Hermeneutik allein in der "Vorselektion von Erklärungshypothesen“ (S. 6) und der (nicht näher spezifizierten) Ökonomisierung des Forschungsprozesses. Hammer (2012) erachtet die Hermeneutik als „Methode der Wahrnehmung und des Verstehens von betrieblichen Sachverhalten“ (S. 33) zwar für relevant, führt diese aber nur sehr vage aus. Helfrich (2016) sieht in der Hermeneutik eine besondere Form der Erklärung, namentlich der funktionalen Erklärung, die ,Sachverhalte in ihrer Beziehung zu übergeordneten Systemen“ betrachtet (S. 25), und die er von einer kausalen Erklärung unterscheidet. Hierbei nimmt er einen systemtheoretischen Blick ein, der auf die Aufdeckung allgemeiner Gesetzmäßigkeiten gerichtet ist. Dabei betont der Autor explizit, dass damit nicht das Erkenntnisziel des Verstehens im oben explizierten Sinne erreicht werden soll, so dass auch hier die naturwissenschaftliche Logik (des Erklärens) nicht verlassen wird. Der Nutzen der Hermeneutik wird entsprechend in der Gewinnung von Hypothesen gesehen. Corsten und Corsten (2019) sehen den hermeneutischen Ansatz als Interpretationsmodelle neben Kausalmodellen als eine alternative Form der Erklärung (S. 14). Dies kommt einer Würdigung des (nicht explizit angeführten) Verstehens noch am nächsten, auch wenn eine erkenntnistheoretische Abgrenzung fehlt. Demgegenüber sieht Bardmann (2019) die Hermeneutik in der Betriebswirtschaftslehre als nicht anerkannte wissenschaftliche Methode an, denn „die hermeneutische Methode ist keine Erklärungsmethode“. Dennoch spricht er der Hermeneutik ,,ihre Berechtigung“ zu, ,wenn es darum geht, Sinnzusammenhänge zu erfassen“" (S. 146). 
Insgesamt zeigt sich deutlich, dass diejenigen Autoren, die die Hermeneutik behandeln, aber das Verstehen nicht als theoretisches Erkenntnisziel neben das Erklären stellen - was lediglich Kirsch et al. (2007) und Schneck (2010) tun -, diese zwar methodisch, nicht aber methodologisch für zulässig erachten. Ansätze des Verstehens sind aus dieser Sicht mit Kieser (1995) gesprochen ,nicht un-, wohl aber vorwissenschaftlich: Sie können zur Bildung von Hypothesen anregen, eine Erklärung mittels Hypothesen jedoch nicht ersetzen“ (S. 21). In der Allgemeinen Betriebswirtschaftslehre gilt auf theoretischer Ebene (fast) ausschließlich die naturwissenschaftliche Logik des Erklärens, während sich eine praktische Relevanz nur auf Basis dieser Logik ergeben kann. Die Möglichkeit eines alternativen Wegs der Erkenntnis durch das geisteswissenschaftliche Verstehen wird (fast) nirgendwo explizit thematisiert. Das Verstehen könnte demnach als Methode zugelassen werden, nicht aber als Methodologie, Epistemologie oder Ontologie. Die Betriebswirtschaftslehre stellt nach gängiger Meinung eine angewandte Geisteswissenschaft mit naturwissenschaftlicher Methodologie dar. Sie folgt einem Methodenmonismus.

Von einer geisteswissenschaftlichen Öffnung der Betriebswirtschaftslehre oder gar einem Paradigmenwechsel kann daher (heute) in der Tat keine Rede sein. Die in den Lehrbüchern vertretene theoretische Fundierung der Betriebswirtschaftslehre stimmt nicht mit der gelebten Forschungspraxis in der Betriebswirtschaftslehre überein. Will sie diese Lücke schließen, muss sie das Verstehen in ihr Fundament integrieren und, als anwendungsorientierte Disziplin, die praktische Relevanz des Verstehens neben dem Erklären würdigen.

\section{Anknüpfungspunkte einer wissenschaftstheoretischen Revision}

Die Betriebswirtschaftslehre deckt den Aspekt der Gestaltung in der Regel durch die Entwicklung technologischen Wissens ab (Kirsch et al. 2007, S. 2). Dieses besitzt instrumentellen Charakter, d. h, es gibt Aufschluss darüber, welche Mittel einzusetzen sind, um ein Ziel zu erreichen (Bunge 2001b, S. 365; Helfrich 2016, S. 3). Für Pfriem (2011) wird die „Reduktion betriebswirtschaftlichen Wissens auf instrumentelles Verfügbarkeitswissen“ sogar ,geradezu unabhängig von dessen Qualität und Aktualität mit Praxisnähe gleichgesetzt“" (S. 288).

Vor diesem Hintergrund ist der Beitrag des Verstehens zur Gestaltung von zwei Seiten herauszuarbeiten. Auf der einen Seite ist zu klären, inwiefern sich erklärende Aussagen tatsächlich in technologische Aussagen transformieren lassen und welche Rolle hierbei das Verstehen spielt. Auf der anderen Seite stellt sich die Frage, inwiefern das Verstehen eine praktische Relevanz besitzt, die sich von der Entwicklung technologischer Aussagen unterscheidet.

\subsection{Zum Verhältnis von Theorie und Technologie}

Die praktische Relevanz des Erklärens wird meist in der Transformation theoretischer in technologische Aussagensysteme gesehen. Im Anschluss an Popper (1949) und Albert (1960) wird die Annahme vertreten, die (theoretische) Prognose und die (praktische) Gestaltung besäßen dieselbe Analyse- und Wirkungsrichtung, weshalb 
Theorie und Technologie strukturell übereinstimmten und von einer tautologischen Transformation gesprochen werden könne (Nienhüser 1989, S. 55 ff.; Seidl et al. 2009, S. 49). Gegen diese Annahme ist allerdings vielfältig Kritik geübt worden.

Im Wesentlichen sind fünf Kritikpunkte an der tautologischen Transformation zu nennen (Helfrich 2016, S. 45; Kirsch et al. 2007, S. 176 ff.; Nienhüser 1989, S. 59 ff.; Seidl et al. 2009, S. 51 ff.). Zunächst weisen Theorien und Technologien einen unterschiedlichen Allgemeinheitsgrad auf, weshalb sich die Allgemeingültigkeit von Theorien nicht ohne weiteres auf den konkreten Handlungsbezug oder Mittelbezug von Technologien übertragen lässt. Grob gesagt sagen Theorien zwar, was zu tun ist, aber nicht wie (also mit welchen Mitteln) etwas erreicht werden kann. Es sind also zusätzliche Informationen notwendig, um von theoretischen zu technologischen Aussagen zu kommen. Zweitens stellen Theorien vereinfachende Abstrahierungen der komplexen Welt dar, die einzelne Aspekte der Realität ausblenden. Da diese in realen Entscheidungssituationen aber faktisch gegeben sein können, lässt sich durch die transformierte Technologie nicht abschließend beurteilen, ob sich mit den postulierten Mitteln auch tatsächlich das gewünschte Ziel erreichen lässt. Drittens fehlt bei einer tautologischen Transformation eine Beurteilung der einzusetzenden Mittel, sowohl in Bezug auf ihre Effizienz im Vergleich mit alternativen Mitteln als auch hinsichtlich ihrer ethisch-normativen Bewertung. Man weiß zwar, mit welchen Mitteln sich ein Zweck erfüllen lässt, aber nicht, ob diese Mittel die effizientesten sind oder eingesetzt werden sollten. Viertens können für eine praktische Entscheidung einander widersprechende Theorien gleichermaßen relevant sein, so dass sich auf technologischer Ebene ein Auswahlproblem ergibt, das nicht auf theoretischer Ebene lösbar ist, während sich auf der anderen Seite nicht für jede praktische Entscheidung eine passende Theorie findet. Fünftens kann von einer tautologischen Transformation nicht zuletzt deshalb keine Rede sein, weil das Kriterium für die Gültigkeit einer erklärenden Aussage von ganz anderer Art ist als für eine technologische Aussage. Für erklärende Aussagen gilt als Kriterium ihr Wahrheitsgehalt, also ihre Übereinstimmung mit der (unabhängigen oder konstruierten) Realität. Demgegenüber gilt für technologische Aussagen als Kriterium ihr praktischer Nutzen im Sinne ihrer Tauglichkeit für zweckgerichtetes Handeln. Theoretische Aussagen sind für eine Entwicklung von Technologien weder notwendig noch hinreichend.

Ähnlich argumentieren auch Sandberg und Tsoukas (2011, S. 341f.) in ihrer grundsätzlichen Kritik an der praktischen Relevanz des Erklärens, die sie als szientistische Rationalität bezeichnen und der praktischen Rationalität (im Sinne einer Logik des Verstehens) gegenüberstellen. Erstens vernachlässigt die Logik des Erklärens den ganzheitlichen Sinnzusammenhang, in den Menschen bei ihren Tätigkeiten verstrickt sind. Potenzielle Lösungen und adäquate Handlungsmöglichkeiten ergeben sich eher aus den mit einer Tätigkeit verbundenen Umständen als aus einem Bündel abstrakter Verfahrensweisen. Zweitens vernachlässigt die Logik des Erklärens die situative Einzigartigkeit, die praktische Probleme aufweisen. Sie generiert Aussagen, die auf einer aggregiert-abstrakten Ebene wahr sein mögen, die konkrete Situation aber zu sehr vereinfachen und dadurch verzerren. Drittens abstrahiert sie das für die Bewältigung von Problemen wesentliche Erleben von Zeit. Der Ablauf von Tätigkeiten, ihre Geschwindigkeit, ihr Rhythmus, ihre Intensität und ihre Rich- 
tung sind für ihren Fluss elementar, werden von der Logik des Erklärens jedoch in vielen Fällen ausgeblendet.

Neben der Problematik der praktischen Transformation oder Relevanz theoretischer Aussagensysteme ergeben sich für das Erklären bereits auf theoretischer Ebene Probleme, die ihre praktische Relevanz weiter einschränken. Dies gilt insbesondere in Bezug auf die epistemologische Annahme, für die soziale Wirklichkeit ließen sich Prognosen analog zu den Naturwissenschaften formulieren. Es ist ein bekanntes Phänomen, dass Prognosen bei ihrer lebensweltlichen Nutzung zu einer selbsterfüllenden Prophezeiung werden und sich auf diese Weise selbst aufheben können. Dies liegt daran, dass Menschen im Gegensatz zu naturwissenschaftlichen Gegenständen auf Vorhersagen reagieren und sich damit die Prämissen der Erklärung ändern. Dies kann dazu führen, dass ein unterstelltes Verhalten erst durch die Unterstellung ausgelöst wird. Sie stellen also mitunter die Realität erst her, die sie eigentlich untersuchen möchten (Aaken et al. 2015, S. 57 f.; Astley 1985, S. 506). Wer etwa glaubhaft einen steigenden Aktienkurs prognostiziert, animiert Anleger dazu, Aktien einzukaufen, wodurch der Aktienkurs steigt (Nicolai und Seidl 2010, S. 1274). Im Gegensatz zu den Erkenntnisgegenständen der Naturwissenschaften ist das menschliche Handeln durch eine Vielzahl interdependenter, sich wandelnder und überdies kulturell geprägter Faktoren bestimmt, so dass sich für dieses keine raumzeitlich invariante Muster ausfindig machen lassen (Aaken et al. 2015, S. 57).

All diese Aspekte sind bei der Anwendung technisch-instrumentellem Verfügungswissen zu berücksichtigen; die ,Vorstellung, ein gegebenes Instrument ließe sich ,schablonenhaft" umsetzen, führt unausweichlich zum Misserfolg“ (Martin und Bartscher-Finzer 2015, S. 29). In einem Klima der Angst lassen sich beispielsweise Feedback-Instrumente nicht effektiv einsetzen, weil sich die Menschen nicht trauen, kritisches Feedback zu geben. Selbst wenn sich durch den Einsatz einer Technologie in der Praxis ein gewünschtes Ziel herbeiführen lässt, können neben den erwünschten Effekten auch unerwünschte Neben-, Rück- oder Folgewirkungen auftreten, die gegebenenfalls nicht einmal wahrgenommen werden. So können z. B. Individualprämien zwar die individuelle Leistung erhöhen, gleichzeitig aber auch die Teambildung und -verantwortung erschweren (Wüthrich 2016, S. 192 f.). Neben der Entwicklung von Technologien wäre daher eine Technik- oder Nebenfolgenabschätzung durchzuführen, die die Wirkungen einer Technologie im konkreten sozialen System untersucht und damit eine konkrete Auseinandersetzung mit deren Dynamiken erfordert. Dies setzt dann aber an der Logik des Verstehens an, da es hierzu erforderlich ist, ,,sich in das soziale Systeme selbst zu begeben, um ein Verständnis für die entsprechenden Vorgänge zu entwickeln“ (Kirsch et al. 2007, S. 208). In diesem Sinne besitzen erklärende Theorien für die Entwicklung von Technologien vor allem eine heuristische Funktion: Sie sensibilisieren den Forscher für bestimmte Zusammenhänge, die ihm bei der Technologieentwicklung als Orientierung dienen (Kirsch et al. 2007, S. 177). Die Wirksamkeit von Technologien hängt entscheidend von einem Verstehen der konkreten Sinnzusammenhänge des sozialen Systems ab, für das und in dem die Technologie entwickelt wird.

Wenn die erklärende Theorie aber nur eine heuristische Funktion bei der Entwicklung von Technologien besitzt, ist die grundsätzliche Frage nach deren Wissenschaftlichkeit zu stellen. Bunge sieht das entscheidende Kriterium der Wissenschaftlichkeit 
einer Technologie in ihrer theoretisch-erklärenden Fundierung. Fehlt diese, spricht er von Pseudotechnologien oder - falls die Entwicklung der Technologie der theoretischen Erklärung vorausgeht - von Prototechnologien (Bunge 1985, S. 231 ff.). Der Hermeneutik spricht Bunge ihre Wissenschaftlichkeit dagegen vollständig ab. Er bezeichnet sie als ,regression to magical thinking“; hermeneutische Studien „,have produced nothing but programmatic utterances, trivialities, and falsities. [...] Therefore, hermeneutic philosophy has nothing to teach social scientists“" (Bunge 2001a, S. 331).

Diesem engen Verständnis der Wissenschaftlichkeit von Technologien - dem hier aufgrund der oben herausgearbeiteten Wissenschaftlichkeit hermeneutischer Ansätze ohnehin nicht gefolgt werden kann - setzen Kirsch et al. (2007) und Seidl et al. (2009) ihren Vorschlag entgegen, die technologische neben der theoretischen Forschung als eigenständigen Forschungsbereich anzuerkennen. Mit ihrer Denkfigur des „Reasoning from Case to Case“ bringen die Autoren zum Ausdruck, dass ein technologischer Forschungsprozess meist allenfalls vorläufige Generalisierungen erlaubt, die es dann fallweise zu modifizieren und weiterzuentwickeln gilt. Für die Betrachtung der jeweiligen Einzelfälle komme es gerade auf Wissensformen an, die nicht auf dem Erklären beruhen, sondern narratives Wissen im Sinne einer Erzählung darstellen. Dadurch ließe sich ein ,wesentlich komplexeres und vielschichtigeres Bild der sozialen ,Wirklichkeit““ (Kirsch et al. 2007, S. 240) nachzeichnen. Als wissenschaftlich im Sinne einer Kunstlehre bezeichnen die Autoren ein solches Reasoning from Case to Case, sofern es systematisch erfolgt. Das Verstehen kommt dabei z.B. im Zuge einer verstehenden Lebensweltanalyse zum Einsatz, mittels derer Forschende die Praktiken einer Organisation rekonstruieren, weshalb die Autoren davon ausgehen, ,dass ein vollständiger [technologischer] Entwicklungsprozess einen direkten Kontakt mit der Unternehmenspraxis impliziert“ (Kirsch et al. 2007, S. 224).

Für eine Betriebswirtschaftslehre, die Erklären und Verstehen als gleichwertig ansieht, wäre das Verhältnis von Theorie und Technologie in dem von Kirsch et al. (2007) und Seidl et al. (2009) aufgezeigten Sinne zu revidieren. Berücksichtigt man die Kritik der Transformation theoretischer in technologische Aussagen, sind die theoretische und die praktische Zielsetzung darüber hinaus als gleichwertig anzusehen. Ansätze, die Theorien (lediglich) als Mittel zur Erreichung praktischer Ziele ansehen, werden weder der theoretischen noch der technologischen Aufgabe gerecht.

\subsection{Verstehen als Orientierungswissen in Entscheidungssituationen}

Da dem geisteswissenschaftlichen Verstehen in der Entwicklung von Technologien traditionell wenig Relevanz zugesprochen wird, findet sich mitunter die Frage, inwiefern die verstehende Wissenschaft der Forderung nach Gestaltung überhaupt gerecht werden kann (Wolf 2013, S. 23). Aus dieser Sicht ergibt sich für das Verstehen eine praktische Relevanz also nur dann, wenn neben dem technologischen Wissen eine alternative Form des praktischen Wissens - und damit eine alternative Form der Gestaltung - existiert, dessen Fundament nicht das Erklären, sondern das Verstehen ist. Eine solche alternative Form des Wissens beinhaltet die von Pfriem (2011) vorgenommene Unterscheidung zwischen technisch-instrumentellem Verfügungswissen auf der einen und Orientierungswissen auf der anderen Seite. Letzteres 
dient als alternative Form des praktischen Wissens ,ganz anders als das technisch-instrumentelle Verfügungswissen dazu, uns in unseren Entscheidungen zu orientieren“ (S. 268).

Damit ergibt sich für das Verstehen eine besondere Form der praktischen Relevanz, die zeitlich und logisch der (Phase der) Mittelwahl vorgeordnet ist: Bevor ein Mittel aus einer gegebenen Menge ausgewählt werden kann, bedarf es zunächst eines Orientierungswissens dahingehend, welche Mittel überhaupt zur Verfügung stehen. Die Orientierung geht dem reinen Wahlakt voraus und unterstützt im Prozess der Entscheidungsfindung die Phasen des Problemerkennens und der Alternativensuche. Menschen können ein Problem verschieden verstehen und entsprechend anders wahrnehmen, handeln und entscheiden. Ähnliches gilt für die Alternativensuche, da Handlungsalternativen nicht ex ante gegeben, sondern aus der Interpretation des Problems expliziert werden müssen (Brodbeck 2014, S. 11 f.). Kirsch et al. (2007) sprechen diesbezüglich von der Problemdefinition oder dem Problem des „Problems“, für das das Verstehen notwendige Voraussetzung ist, denn das „Problem kann nur verstanden werden, wenn sich der Forscher als Teilnehmer dieser Lebens-, Sprach- und Wissensform ein ,Know-how “ der sie konstituierenden Regeln und Weltanschauungen aneignet" (S. 191). Für die Autoren besitzt bereits die Explikation des Problems als wissenschaftliches Ergebnis - also ohne die Entwicklung von Technologien - eine praktische Relevanz und ist Ausfluss einer anwendungsorientierten Betriebswirtschaftslehre:

Exakte und fruchtbare Problemdefinitionen können für die Praxis große Bedeutung besitzen, auch wenn sie bei der Lösung dieser Probleme zunächst sich selbst überlassen bleiben muss, weil die Betriebswirtschaftslehre noch keine adäquaten Methoden zur Unterstützung dieser Problemlösungsbemühungen kennt. (Kirsch et al. 2007, S. 199)

Ähnlich unterscheiden Nicolai und Seidl (2010) mit instrumentellem und konzeptionellem Wissen zwei verschiedene Wissensformen mit grundverschiedener Art der praktischen Relevanz. Instrumentelles Wissen stellt Handlungswissen (im Sinne eines ,knowledge for action“, S. 1264) dar und bezieht sich auf Kenntnisse darüber, mit welchen Mitteln sich welche Zwecke erreichen lassen. Im Wesentlichen handelt es sich also um technisch-instrumentelles Verfügungswissen. Die Autoren legen dar, dass das Verstehen für die Anwendung dieses Wissens keine notwendige Voraussetzung ist:

The three types of instrumental knowledge discussed here typically require no understanding of the larger context within which that knowledge was developed. This is what the concept of ,instrument ' implies: in order to use an instrument, all one needs to know is under what conditions it is to be used and what effect it has. One might have to specify given variables on the basis of contextspecific information, or calibrate the instrument, but one need not understand how and why the instrument works. This is true for schemes and forecasts but also for technological rules. (Nicolai und Seidl 2010, S. 1276)

Demgegenüber bezieht sich konzeptionelles Wissen auf Kenntnisse bezüglich der Definition der Entscheidungssituation (im Sinne eines „,knowledge for understand- 
ing“, S. 1264) bzw. der Problemdefinition nach Kirsch et al. (2007). Es handelt sich um Orientierungswissen, das ein Verstehen notwendig voraussetzt:

In the case of conceptual forms of knowledge, the situation is very different. They usually require an-at least rudimentary — understanding of the theoretical context in which that knowledge is embedded. Without that understanding, the meaning of those forms of knowledge cannot be grasped. In contrast to instrumental knowledge, where it is enough to understand how to use it, the main characteristic of conceptual forms of knowledge is that they broaden our understanding. (Nicolai und Seidl 2010, S. 1276)

Für die Autoren ergibt sich die praktische Relevanz beider Wissensformen jeweils für verschiedene Phasen im Prozess der Entscheidungsfindung. Konzeptionelles Wissen unterstützt den/die Entscheider*in bei der Definition der Entscheidungssituation, instrumentelles Wissen in der anschließenden Auswahl einer Alternative. Für die Definition der Entscheidungssituation ist verstehende Forschung demzufolge in dem Maße praktisch relevant, in dem sie die Wahrnehmung bzw. Konstruktion von realen Entscheidungssituationen bzw. die dahinterstehenden mentalen Modelle beeinflusst. Konzeptionelles Wissen entsteht z. B. als Orientierungswissen, wenn eine Theorie gängiges Wissen infrage stellt und so eine neue bzw. reichhaltigere Perspektive auf die Entscheidungssituation ermöglicht. Während instrumentelles Wissen zudem einen ,one-best-way“ suggeriert, kann konzeptionelles Wissen auch einander widersprechende Theorien integrieren, indem diese in ein dialektisches Verhältnis zueinander gebracht werden und so ein umfassenderes Problembewusstsein anregen. Diese konzeptionelle Integrationsleistung ist ohne ein Verstehen nicht möglich. Sie folgt einer Rationalität, die Sandberg und Tsoukas (2011, S. 352 f.) praktische Rationalität nennen und die sie in Heideggers Theorie des In-der-Welt-Seins einbetten, nach der das (immer schon gegebene und allenfalls hermeneutisch zu erschließende) Verstrickt-Sein des Menschen seine Lebenswelt fundiert (Heidegger 2006). Aus Sicht einer praktischen Rationalität sind Theorien teilweise emergente Kreationen, die den Menschen bei seiner Orientierung in der Welt unterstützen, dabei aber stets interpretationsoffen bleiben. Anstatt die Mittel zur Erreichung von Zielen explizit vorzugeben, ermöglicht gerade die Unabgeschlossenheit eine situationsspezifische Auslegung.

Als Beispiel für praktisch relevantes Orientierungswissen lassen sich mit Pfriem die 5 P's (plan, ploy, pattern, position und perspective) von Mintzberg (1987) anführen, die jeweils eine unterschiedliche Facette strategischer Entscheidungen betonen:

Plan, Spielzug, Handlungsmuster und Selbstverortung sind eingewoben in die Frage der Perspektive, bei der es darum geht, wie die Unternehmung sich und ihre Umweltbeziehungen eigentlich konstruiert. Das hat mit kontingenten, also möglichen, unterschiedlich denkbaren Deutungen und sinnhaften Orientierungen zu tun, die auf die Welt, sprich die Umwelten und ihre inhaltlich-materiellen Herausforderungen zu beziehen sind. (Pfriem 2011, S. 304)

Es geht also nicht darum, eine neue Form des Wissens in die Betriebswirtschaftslehre einzuführen, sondern die praktische Relevanz von Orientierungswissen - z. B. in einem Lehrbuch zur Allgemeinen Betriebswirtschaftslehre - transparent zu ma- 
chen und in ihren Implikationen von der grundsätzlich anders gelagerten praktischen Relevanz technisch-instrumentellen Verfügungswissens abzugrenzen.

\section{Fazit}

Im Ergebnis lässt sich festhalten: Die Betriebswirtschaftslehre versteht sich überwiegend noch immer (oder wieder) als anwendungsorientierte Geisteswissenschaft mit naturwissenschaftlichem Forschungsideal. Die Verengung der Disziplin auf das Erklären (Methodenmonismus) ist vor allem dahingehend problematisch, dass sie nicht mit der praktizierten Wissenschaft übereinstimmt (Methodenpluralismus). Dieser „Double Talk“ zwischen methodologischer Verengung und Offenheit erzeugt nicht nur eine diffuse Gemengelage in Bezug auf die Frage, was die Betriebswirtschaftslehre gelten lassen will - er kann sich auch zu einem „Double Bind“ entwickeln. Für qualitativ forschende Nachwuchswissenschaftler*innen kann dieser etwa dadurch entstehen, dass in ihrem Forschungsdesign zwar kein Problem gesehen wird, Möglichkeiten der Anwendungsorientierung aber ausschließlich in der Ableitung allgemeingültiger Ziel-Mittel-Aussagen gesehen werden.

Für eine anwendungsorientierte Wissenschaft wie die Betriebswirtschaftslehre wäre es aber verkürzt, das Verstehen lediglich als theoretische Aufgabe ohne Anwendungsbezug zu betrachten. Ebenso ist ein potenzieller Beitrag verstehender Forschung aufzuzeigen, dem Menschen in betrieblichen Kontexten zu einer besseren Lebensbewältigung zu verhelfen. Dieser Beitrag sieht die praktische Relevanz des Verstehens zum einen in der Entwicklung technologischen Wissens und zum anderen in der Vermittlung von Orientierungswissen, das im Gegensatz zum technischinstrumentellen Verfügungswissen keine konkreten Mittel zur Zielerreichung suggeriert, sondern - diesem Schritt vorgelagert - den Menschen bei der Definition der Entscheidungssituation unterstützt. Von dieser ausgehend kann er dann entweder unmittelbar selbst eine Entscheidung treffen oder auf technisch-instrumentelles Verfügungswissen zurückgreifen, um eine passende Alternative auszuwählen. Eine anwendungsorientierte Betriebswirtschaftslehre kann nicht alleine darauf abstellen, (sozial-)technologische Gestaltungsempfehlungen zu geben. Sie muss darüber hinaus Orientierungswissen bereitstellen, das dem Menschen in betrieblichen Kontexten hilft, seine Entscheidungssituation zu verstehen und aus diesem Verständnis heraus eine angemessene Entscheidung treffen zu können - mit oder ohne Technologien.

Funding Open Access funding provided by Projekt DEAL.

Open Access Dieser Artikel wird unter der Creative Commons Namensnennung 4.0 International Lizenz veröffentlicht, welche die Nutzung, Vervielfältigung, Bearbeitung, Verbreitung und Wiedergabe in jeglichem Medium und Format erlaubt, sofern Sie den/die ursprünglichen Autor(en) und die Quelle ordnungsgemäß nennen, einen Link zur Creative Commons Lizenz beifügen und angeben, ob Änderungen vorgenommen wurden.

Die in diesem Artikel enthaltenen Bilder und sonstiges Drittmaterial unterliegen ebenfalls der genannten Creative Commons Lizenz, sofern sich aus der Abbildungslegende nichts anderes ergibt. Sofern das betreffende Material nicht unter der genannten Creative Commons Lizenz steht und die betreffende Handlung 
nicht nach gesetzlichen Vorschriften erlaubt ist, ist für die oben aufgeführten Weiterverwendungen des Materials die Einwilligung des jeweiligen Rechteinhabers einzuholen.

Weitere Details zur Lizenz entnehmen Sie bitte der Lizenzinformation auf http://creativecommons.org/ licenses/by/4.0/deed.de.

Interessenkonflikt C. Julmi gibt an, dass kein Interessenkonflikt besteht.

\section{Literatur}

van Aaken, Dominik, Werner Kirsch, und David Seidl. 2015. Gesetzmäßigkeiten in der Unternehmensführung? Unternehmensführung als das Verfügen über Notwendigkeiten. Die Unternehmung 69(1):54-66.

Albert, Hans. 1960. Wissenschaft und Politik: Zum Problem der Anwendbarkeit einer wertfreien Sozialwissenschaft. In Probleme der Wissenschaftstheorie, Hrsg. Ernst Topitsch, 201-232. Berlin: Springer.

Amely, Tobias, und Thomas Krickhahn. 2016. Bwl für Dummies, 3. Aufl., Weinheim: Wiley.*1

Antoni, Manfred. 1983. Vor einem Paradigmawechsel? Betriebswirtschaftslehre als Kulturwissenschaft. In Paradigmawechsel in der Betriebswirtschaftslehre?, Hrsg. Wolf F. Fischer-Winkelmann, 54-78. Spardorf: Wilfer.

Arnold, Bernd. 2016. Betriebswirtschaft kompakt. Stuttgart: EduMedia.*

Astley, W. Graham 1985. Administrative science as socially constructed truth. Administrative Science Quarterly 30(4):497-513.

Backhaus, Klaus, Uta Herbst, Markus Voeth, und Robert Wilken. 2010. Allgemeine Betriebswirtschaftslehre: Koordination betrieblicher Entscheidungen, 4. Aufl., Berlin: Springer.*

Balderjahn, Ingo, und Günter Specht. 2016. Einführung in die Betriebswirtschaftslehre, 7. Aufl., Stuttgart: Schäffer-Poeschel.*

Bardmann, Manfred. 2019. Grundlagen der Allgemeinen Betriebswirtschaftslehre: Geschichte - Konzepte - Digitalisierung, 3. Aufl., Wiesbaden: Springer Gabler.*

Bernecker, Michael. 2009. Grundlagen der Betriebswirtschaftslehre, 3. Aufl., Köln: johanna Verlag.*

Beschorner, Dieter, und Volker H. Peemöller. 2006. Allgemeine Betriebswirtschaftslehre: Grundlagen und Konzepte, 2. Aufl., Herne: Neue Wirtschafts-Briefe.*

Birker, Klaus. 2006. Einführung in die Betriebswirtschaftslehre: Grundbegriffe, Denkweisen, Fachgebiete, 2. Aufl., Berlin: Cornelsen.*

Bogdan, Robert, und Sari Knopp Biklen. 2007. Qualitative research for education: an introduction to theories and methods, 5. Aufl., New York: Pearson.

Brockhoff, Klaus. 2017. Betriebswirtschaftslehre in Wissenschaft und Geschichte: Eine Skizze, 5. Aufl., Wiesbaden: Springer Gabler.

Brodbeck, Karl-Heinz. 2014. Die fragwürdigen Grundlagen der Ökonomie: Eine philosophische Kritik der modernen Wirtschaftswissenschaften, 6. Aufl., Darmstadt: WBG (Wissenschaftliche Buchgesellschaft).

Bunge, Mario. 1985. Epistemology \& Methodology III: Philosophy of science and technology: Part II: Life science, social science and technology. Treatise on basic philosophy, Bd. 7. Dordrecht: Reidel.

Bunge, Mario. 2001a. Realism and antirealism in social science. In Scientific realism: selected essays of Mario Bunge, Hrsg. Martin Mahner, 320-342. Amherst: Prometheus Books.

Bunge, Mario. 2001b. The technologies in philosophy. In Scientific realism: selected essays of Mario Bunge, Hrsg. Martin Mahner, 365-374. Amherst: Prometheus Books.

Burrell, Gibson, und Gareth Morgan. 1979. Sociological paradigms and organisational analysis. Elements of the sociology of corporate life. London: Heinemann.

Chmielewicz, Klaus. 1970. Forschungskonzeption der Wirtschaftswissenschaft. Stuttgart: Poeschel.

Cohen, Louis, Lawrence Manion, und Keith Morrison. 2018. Research methods in education, 8. Aufl., London: Routledge.

Corsten, Martina, und Hans Corsten. 2019. Betriebswirtschaftslehre, 2. Aufl., München: Vahlen.*

Creswell, John W., und J. David Creswell. 2018. Research design: qualitative, quantitative, and mixed methods approaches, 5. Aufl., Los Angeles et al: SAGE.

Deelmann, Thomas. 2015. Meilensteine und Trends der Betriebswirtschaft: Grundlagen, Geschichte und Geschichten der BWL, 2. Aufl., Berlin: Schmidt.*

\footnotetext{
${ }^{1}$ Die mit einem Sternchen markierten Quellen waren Teil des Reviews.
} 
Deutsche Nationalbibliothek. 2019. Katalog der Deutschen Nationalbibliothek. portal.dnb.de.

Dilthey, Wilhelm. 1924. Gesammelte Schriften. V. Band: Die geistige Welt: Einleitung in die Philosophie des Lebens. Erste Hälfte. Leipzig: Teubner.

Domschke, Wolfgang, und Armin Scholl. 2008. Grundlagen der Betriebswirtschaftslehre: Eine Einführung aus entscheidungsorientierter Sicht, 4. Aufl., Berlin: Springer.*

Egger, Anton, Walter Egger, und Reinbert Schauer. 2016. Einführung in die Allgemeine Betriebswirtschaftslehre, 27. Aufl., Wien: Linde.*

Eichhorn, Peter, und Joachim Merk. 2016. Das Prinzip Wirtschaftlichkeit: Basiswissen der Betriebswirtschaftslehre, 4. Aufl., Wiesbaden: Springer Gabler**

Eisend, Martin, und Alfred Kuß. 2017. Grundlagen empirischer Forschung: Zur Methodologie in der Betriebswirtschaftslehre. Wiesbaden: Springer Gabler.

Erdmann, Georg, und Michael Krupp. 2018. Betriebswirtschaftslehre. Hallbergmoos: Pearson.*

Fischer-Winkelmann, Wolf F. (Hrsg.). 1983. Paradigmawechsel in der Betriebswirtschaftslehre? Spardorf: Wilfer.

Geyer, Helmut, und Bernd Ahrendt. 2016. Crashkurs BWL, 6. Aufl., Freiburg: Haufe.*

Groß, Steffen W. 2019. Weder rein nomothetisch noch allein idiographisch: Philosophische Gründe für die Bestimmung der Ökonomie als Kulturwissenschaft. In Spiel-Räume des Denkens: Festschrift zu Ehren von Karl-Heinz Brodbeck, Hrsg. Silja Graupe, Walter Otto Ötsch, und Florian Rommel, 181-218. Marburg: Metropolis.

Hammer, Richard M. 2012. Führungsorientierte Betriebswirtschaftslehre, 2. Aufl., Wien: Manz.*

Harbrücker, Ulrich. 2008. Einführung in die Betriebswirtschaftslehre, 2. Aufl., Altenberge: Niederle-Media.*

Heidegger, Martin. 2006. Sein und Zeit, 19. Aufl., Tübingen: Max Niemeyer Verlag.

Heise, Arne. 2015. Aus dem Gleichgewicht. Über den Zustand der Wirtschaftswissenschaften. Forschung \& Lehre 22(5):376-377.

Helfrich, Hede. 2016. Wissenschaftstheorie für Betriebswirtschaftler. Wiesbaden: Springer Gabler.*

Hitzler, Ronald, und Thomas S. Eberle. 2017. Phänomenologische Lebensweltanalyse. In Qualitative Forschung, 12. Aufl., Hrsg. Uwe Flick, Ernst von Kardorff, und Ines Steinke, 109-118. Reinbek: Rowohlt.

Hummel, Thomas R. 2007. Betriebswirtschaftslehre kompakt, 3. Aufl., München: Oldenbourg.*

Hutzschenreuter, Thomas. 2015. Allgemeine Betriebswirtschaftslehre: Grundlagen mit zahlreichen Praxisbeispielen, 6. Aufl., Wiesbaden: Springer Gabler.

Julmi, Christian. 2019. Wissenschaftstheoretische Grundlagen einer anwendungsorientierten postmechanischen Ökonomie. In Spiel-Räume des Denkens: Festschrift zu Ehren von Karl-Heinz Brodbeck, Hrsg. Silja Graupe, Walter Otto Ötsch, und Florian Rommel, 237-256. Marburg: Metropolis.

Jung, Hans. 2016. Allgemeine Betriebswirtschaftslehre, 13. Aufl., Berlin: De Gruyter.*

von Känel, Siegfried. 2018. Betriebswirtschaftslehre: Eine Einführung. Wiesbaden: Springer Gabler.*

Kieser, Alfred. 1995. Anleitung zum kritischen Umgang mit Organisationstheorien. In Organisationstheorien, 2. Aufl., Hrsg. Alfred Kieser, 1-30. Stuttgart: Kohlhammer.

Kirsch, Werner. 1997. Betriebswirtschaftslehre: Eine Annäherung aus der Perspektive der Unternehmensführung, 4. Aufl., München: Verlag Barbara Kirsch.

Kirsch, Werner, David Seidl, und Dominik van Aaken. 2007. Betriebswirtschaftliche Forschung. Wissenschaftstheoretische Grundlagen und Anwendungsorientierung. Stuttgart: Schäffer-Poeschel.*

Klockhaus, Heinz-E. 2012. BWL für Ahnungslose. Stuttgart: S. Hirzel Verlag.*

Kocian-Dirr, Claudia. 2019. Betriebswirtschaftslehre - Schnell erfasst. Berlin: Springer Gabler.*

Kornmeier, Martin. 2007. Wissenschaftstheorie und wissenschaftliches Arbeiten. Eine Einführung für Wirtschaftswissenschaftler. Heidelberg: Physica-Verlag.*

Kreuzer, Christian. 2019. BWL kompakt: Die 100 wichtigsten Themen der Betriebswirtschaft für Praktiker, 5. Aufl., Wien: Linde.*

Kromphardt, Jürgen, Peter Clever, und Heinz Klippert. 1979. Methoden der Wirtschafts- und Sozialwissenschaften: Eine wissenschaftskritische Einführung. Wiesbaden: Gabler.

Kuhlmann, Annette M., und Werner Sauter. 2009. Allgemeine Betriebswirtschaftslehre. Berlin: TEIA.*

Kußmaul, Heinz. 2016. Betriebswirtschaftslehre: Eine Einführung für Einsteiger und Existenzgründer, 8. Aufl., Berlin: De Gruyter.*

Lamprea, Pedro J. 2009. BWL verstehen: Betriebswirtschaftliche Zusammenhänge in der Praxis. Berlin: Cornelsen.*

Lange, Burkhard. 2018. Grundlagen der Betriebswirtschaftslehre. Aachen: Shaker.*

Martin, Albert, und Susanne Bartscher-Finzer. 2015. Personal: Sozialisation, Integration, Kontrolle. Stuttgart: Kohlhammer. 
Mentzel, Klaus. 2013. Basiswissen Betriebswirtschaftslehre. Dortmund: W3L-Verlag.*

Mentzel, Wolfgang. 2015. BWL Grundwissen, 6. Aufl., Freiburg: Haufe.*

Messner, Stephanie, Christian Kreidl, und Thomas Wala. 2016. Grundlagen der Betriebswirtschaftslehre, 5. Aufl., Wien: LexisNexis.*

Mihalic, Victor. 2012. ABC der Betriebswirtschaft: Die etwas andere Einführung in die Welt betriebswirtschaftlicher Grundlagen und unternehmerischen Denkens, 8. Aufl., Wien: Linde.*

Mintzberg, Henry. 1987. The strategy concept I: Five Ps for strategy. California Management Review 30(1):11-24.

Mülder, Wilhelm, und Daniel Lorberg. 2015. Grundlagen der Betriebswirtschaftslehre. Herne: NWB Verlag.*

Nicolai, Alexander T., und David Seidl. 2010. That's relevant! Different forms of practical relevance in management science. Organization Studies 31(9/10):1257-1285.

Nienhüser, Werner. 1989. Die praktische Nutzung theoretischer Erkenntnisse in der Betriebswirtschaftslehre: Probleme der Entwicklung und Prüfung technologischer Aussagen. Stuttgart: Poeschel.

Olfert, Klaus, und Horst-Joachim Rahn. 2017. Einführung in die Betriebswirtschaftslehre, 12. Aufl., Herne: Kiehl.*

Opresnik, Marc Oliver, und Carsten Rennhak. 2015. Allgemeine Betriebswirtschaftslehre: Grundlagen unternehmerischer Funktionen, 2. Aufl., Berlin: Springer Gabler.*

Paul, Joachim. 2015. Praxisorientierte Einführung in die Allgemeine Betriebswirtschaftslehre, 3. Aufl., Wiesbaden: Springer Gabler.*

Pepels, Werner. 2014. Einführung in die allgemeine Betriebswirtschafts- und Managementlehre, 2. Aufl., Berlin: Berliner Wissenschafts-Verlag.

Pepels, Werner. 2017. Handbuch der Betriebswirtschaft: Das moderne Wissen über Konzept und erfolgreiche Steuerung von Einzelwirtschaften. Berlin: Duncker \& Humblot.*

Peters, Sönke, Rolf Brühl, und Johannes N. Stelling. 2005. Betriebswirtschaftslehre, 12. Aufl., München: Oldenbourg.*

Pfriem, Reinhard. 2011. Heranführung an die Betriebswirtschaftslehre, 3. Aufl., Marburg: Metropolis.

Pietsch, Gotthard. 2015. Opportunismus und Sensemaking in der Unternehmenskrise. Berlin: Logos.

Pilz, Gerald. 2017. Grundwissen BWL, 2. Aufl., Berlin: UVK.*

Popper, Karl R. 1949. Naturgesetze und theoretische Systeme. In Gesetz und Wirklichkeit, Hrsg. Simon Moser, 43-60. Innsbruck: Tyrolia.

Sandberg, Jörgen, und Haridimos Tsoukas. 2011. Grasping the logic of practice: theorizing through practical rationality. Academy of Management Review 36(2):338-360.

Schäffer, Christoph. 2015. Grundlagen betriebswirtschaftlicher Unternehmensführung: Basiswissen für die Managementpraxis, 2. Aufl., Wien: Manz.*

Schanz, Günther. 2018. Eine kurze Geschichte der Betriebswirtschaftslehre, 2. Aufl., Berlin: UVK.

Schauer, Reinbert. 2019. Betriebswirtschaftslehre: Grundlagen, 6. Aufl., Wien: Linde.*

Scherer, Andreas G. 1998. Pluralism and incommensurability in strategic management and organization theory: a problem in search of a solution. Organization 5(2):147-168.

Scherer, Andreas G., und Emilio Marti. 2019. Wissenschaftstheorie der Organisationstheorie. In Organisationstheorien, 8. Aufl., Hrsg. Alfred Kieser, Mark Ebers, 17-44. Stuttgart: Kohlhammer.

Schierenbeck, Henner, und Claudia B. Wöhle. 2016. Grundzüge der Betriebswirtschaftslehre, 19. Aufl., Berlin: De Gruyter.*

Schmalen, Helmut, und Hans Pechtl. 2013. Grundlagen und Probleme der Betriebswirtschaft, 15. Aufl., Stuttgart: Schäffer-Poeschel.*

Schneck, Ottmar. 2010. BWL Basiswissen: Studienausgabe, 2. Aufl., Weinheim: Wiley.*

Schulte, Reinhard. 2007. Betriebswirtschaftslehre: Eine konzeptionelle Einführung unter besonderer Berücksichtigung der Unternehmensgründung. Aachen: Shaker.*

Schultz, Volker. 2016. BWL Basiswissen: Ein Schnellkurs für Nicht-Betriebswirte, 3. Aufl., München: C.H. Beck.*

Schultze, Ulrike, und Charles Stabell. 2004. Knowing what you don't know? Discourses and contradictions in knowledge management research. Journal of Management Studies 41(4):549-573.

Schweitzer, Marcell, und Alexander Baumeister. 2015. Allgemeine Betriebswirtschaftslehre: Theorie und Politik des Wirtschaftens in Unternehmen, 11. Aufl., Berlin: Erich Schmidt.*

Seidl, David, Werner Kirsch, und Dominik van Aaken. 2009. Zur Methodologie der technologischen Forschung in der Betriebswirtschaftslehre. In Methoden in der Betriebswirtschaftslehre, Hrsg. Andreas Georg Scherer, Ina Maria Kaufmann, und Moritz Patzer, 47-69. Wiesbaden: Gabler.

Sigloch, Jochen, Thomas Egner, und Stephan Wildner. 2015. Einführung in die Betriebswirtschaftslehre, 5. Aufl., Stuttgart: Kohlhammer.* 
Soeffner, Hans-Georg. 2017. Sozialwissenschaftliche Hermeneutik. In Qualitative Forschung, 12. Aufl., Hrsg. Uwe Flick, Ernst von Kardorff, und Ines Steinke, 164-175. Reinbek: Rowohlt.

Steinmann, Horst. 1978. Die Betriebswirtschaftslehre als normative Handlungswissenschaft. In Betriebswirtschaftslehre als normative Handlungswissenschaft, Hrsg. Horst Steinmann, 73-103. Wiesbaden: Gabler.

Steinmann, Horst, Hans Böhm, Wolfram Braun, Elmar Gerum, und Georg Schreyögg. 1976. Betriebswirtschaftslehre und Praxis: Vorüberlegungen auf der Grundlage der konstruktiven Philosophie und Wissenschaftstheorie. In Zum Praxisbezug der Betriebswirtschaftslehre in wissenschaftstheoretischer Sicht, Hrsg. Hans Ulrich, 51-92. Bern: Haupt.

Straub, Thomas. 2015. Einführung in die allgemeine Betriebswirtschaftslehre, 2. Aufl., Hallbergmoos: Pearson.*

Strübing, Jörg. 2018. Qualitative Sozialforschung: Eine komprimierte Einführung, 2. Aufl., Berlin: De Gruyter Oldenbourg.

Sturm, Rüdiger. 2006. Allgemeine Betriebswirtschaftslehre. München: Oldenbourg.*

Teuscher, Heinz. 2011. Betriebswirtschaft: Einführung in die Problemstellungen und Lösungskonzepte der Betriebswirtschaftslehre, 2. Aufl., Zürich: Compendio Bildungsmedien.*

Thommen, Jean-Paul, Ann-Kristin Dirk Ulrich Gilbert Achleitner, Dirk Hachmeister, und Gernot Kaiser. 2017. Allgemeine Betriebswirtschaftslehre, 8. Aufl., Wiesbaden: Springer Gabler.*

Töpfer, Armin. 2007. Betriebswirtschaftslehre: Anwendungs- und prozessorientierte Grundlagen, 2. Aufl., Berlin: Springer**

Vahs, Dietmar, und Jan Schäfer-Kunz. 2015. Einfïhrung in die Betriebswirtschaftslehre, 7. Aufl., Stuttgart: Schäffer-Poeschel.*

Vettiger, Hans. 2006. Einführung in die Betriebswirtschafts- und Managementlehre: Begriffe, Konzepte und aktuelle Beispiele. Chur: Rüegger.*

Voss, Rödiger. 2018. BWL kompakt: Grundwissen Betriebswirtschaftslehre, 8. Aufl., Rinteln: Merkur.*

Weber, Wolfgang. 2018. Allgemeine versus Spezielle Betriebswirtschaftslehre. In Ideengeschichte der $B W L$, Hrsg. Wenzel Matiaske, Wolfgang Weber, 21-39. Wiesbaden: Springer.

Weber, Wolfgang, Rüdiger Kabst, und Matthias Baum. 2018. Einführung in die Betriebswirtschaftslehre, 10. Aufl., Wiesbaden: Springer Gabler**

Wehrlin, Ulrich. 2014. Grundlagen der Betriebswirtschaftslehre, 2. Aufl., Göttingen: Optimus.*

Weik, Elke. 2005. Ansätze des Verstehens, Hermeneutik und Phänomenologie. In Handlungsorientierte Ansätze, 2. Aufl., Moderne Organisationstheorien 1., Hrsg. Elke Weik, Lang Rainhart, 96-120. Wiesbaden: Gabler.

Wettengl, Steffen. 2018. Einführung in die Betriebswirtschaftslehre. Weinheim: Wiley.*

Wiltinger, Angelika, und Kai Wiltinger. 2005. Einführung in die Betriebswirtschaft: Systematische Darstellung in Übersichten. Göttingen: Cuvillier.*

Wöhe, Günter, Ulrich Döring, und Gerrit Brösel. 2016. Einführung in die allgemeine Betriebswirtschaftslehre, 26. Aufl., München: Vahlen.*

Wolf, Joachim. 2013. Organisation, Management, Unternehmensführung. Theorien, Praxisbeispiele und Kritik, 5. Aufl., Wiesbaden: Springer Gabler.

Wüthrich, Hans A. 2016. Vorsicht, Management! Beipackzettel beachten. Zeitschrift Führung + Organisation 85(3):192-194. 